\title{
Revealing the Role of Metals and Anions in Nitrophenol UV-Vis. Spectroscopies and their Atmospheric Implication
}

Hailiang Zhao ${ }^{1,2}$, Xiaotong Jiang ${ }^{3}$, Dandan Han $^{2}$, Yaming Sun ${ }^{1}$, Yuhao Liu ${ }^{1}$, Xue Song ${ }^{1}$, and Xia Sheng ${ }^{2 *}$

${ }^{1}$ School of Environmental Engineering, Henan University of Technology, Lianhua Street 100, 450001 Zhengzhou, China

${ }^{2}$ College of Sciences, Henan Agricultural University, Nongye Road 63, 450002 Zhengzhou, China

${ }^{3}$ School of Earth Sciences, Zhejiang University, Zheda Road 38, 310027 Hangzhou, China

e-mail: shengxia@henau.edu.cn 
Table S1. Selected parameters of the $p$-nitrophenol-containing systems obtained at the B3LYP-D3/def2-TZVP level

\begin{tabular}{|c|c|c|c|c|c|}
\hline Complex & $\Delta \mathrm{E}_{\mathrm{INT}}{ }^{a}$ & $\Delta G_{298 K}^{\theta}$ & $\Delta \tilde{v}^{\mathrm{b}}$ & $\Delta \mathrm{r}^{\mathrm{c}}$ & $\Delta \theta^{\mathrm{d}}$ \\
\hline $\mathrm{Mg}^{2+}-p$-nitrophenol (a) & -426.7 & & & & \\
\hline $\mathrm{Mg}^{2+}-p$-nitrophenol (b) & -690.2 & & & & \\
\hline $\mathrm{Mg}^{2+}-p$-nitrophenol (c) & -543.6 & & 114 & 0.010 & -10.9 \\
\hline $\mathrm{Mg}^{2+}-p$-nitrophenol (d) & -440.5 & & & & \\
\hline $\mathrm{Ca}^{2+}-p$-nitrophenol (b) & -503.3 & & & & \\
\hline $\mathrm{Ca}^{2+}-p$-nitrophenol (d) & -279.1 & & 96.9 & 0.008 & -9.8 \\
\hline $\mathrm{Na}^{+}-p$-nitrophenol (b) & -168.1 & & & & \\
\hline $\mathrm{Na}^{+}-p$-nitrophenol (d) & -68.3 & & 28 & 0.003 & -4.6 \\
\hline $\mathrm{K}^{+}-p$-nitrophenol (b) & -128.4 & & & & \\
\hline $\mathrm{K}^{+}-p$-nitrophenol (d) & -42.7 & & 22 & 0.002 & -3.5 \\
\hline$p$-nitrophenol- $-\mathrm{NO}_{3}^{-}$ & -148.2 & & 1271 & 0.070 & -1.6 \\
\hline$p$-nitrophenol- $\mathrm{Cl}^{-}$ & -174.8 & & 1385 & 0.079 & -1.7 \\
\hline $\mathrm{Mg}^{2+}-p$-nitrophenol- $\mathrm{NO}_{3}^{-}$ & -1431.6 & -1396.6 & 400 & 0.835 & -12.0 \\
\hline $\mathrm{Mg}^{2+}-p$-nitrophenol- $\mathrm{Cl}^{-}$ & -1510.5 & -1453.5 & 1022 & 1.039 & -12.0 \\
\hline $\mathrm{Ca}^{2+}-p$-nitrophenol- $\mathrm{NO}_{3}^{-}$ & -1204.0 & -1168.5 & 453 & 0.804 & -11.6 \\
\hline $\mathrm{Ca}^{2+}-p$-nitrophenol- $\mathrm{Cl}^{-}$ & -1281.3 & -1223.9 & 1071 & 0.979 & -11.6 \\
\hline $\mathrm{Na}^{+}-p$-nitrophenol- $\mathrm{NO}_{3}{ }^{-}$ & -530.4 & -494.1 & 1313 & 0.534 & -5.9 \\
\hline $\mathrm{Na}^{+}-p$-nitrophenol- $\mathrm{Cl}^{-}$ & -598.0 & -540.0 & 2008 & 0.538 & -6.0 \\
\hline $\mathrm{K}^{+}-p$-nitrophenol- $\mathrm{NO}_{3}^{-}$ & -465.1 & -429.9 & 1165 & 0.566 & -6.7 \\
\hline $\mathrm{K}^{+}-p$-nitrophenol- $\mathrm{Cl}^{-}$ & -532.2 & -475.2 & 1824 & 0.604 & -6.7 \\
\hline
\end{tabular}

Bond lengths are in $\AA$; frequencies are in $\mathrm{cm}^{-1}$; bond angles are in degrees. ${ }^{a} \Delta E_{I N T}$ corrected with ZPVE. ${ }^{b} \Delta \tilde{v}=\tilde{v}_{\text {monomer }}-\tilde{v}_{\text {complex }}$, is the change in the $\mathrm{OH}-$ stretching transition frequencies upon complexation. ${ }^{d} \Delta r=r_{\text {complex }}-r_{\text {monomer }}$, is the change in the $\mathrm{OH}$ bond length upon complexation. ${ }^{\mathrm{d}} \Delta \theta=\theta_{\text {complex }}-\theta_{\text {monomer, }}$, is the change in the ONO angle upon complexation. 
Table S2. Selected parameters of the deprotonated $p$-nitrophenol-containing systems obtained at the B3LYP-D3/def2-TZVP level

\begin{tabular}{ccc}
\hline Complex & $\mathrm{d}^{\mathrm{a}}$ & $\Delta \theta^{\mathrm{b}}$ \\
\hline $\mathrm{Mg}^{2+}-p$-nitrophenol- (a) & 1.806 & 4.6 \\
$\mathrm{Mg}^{2+}-p$-nitrophenol- (b) & -- & -9.0 \\
$\mathrm{Ca}^{2+}-p$-nitrophenol- (a) & 1.921 & 4.0 \\
$\mathrm{Ca}^{2+}-p$-nitrophenol- (b) & -- & -8.7 \\
$\mathrm{Na}^{+}-p$-nitrophenol- (a) & 1.986 & 1.9 \\
$\mathrm{Na}^{+}-p$-nitrophenol- (b) & -- & -4.0 \\
$\mathrm{~K}^{+}-p$-nitrophenol- (a) & 2.313 & 1.8 \\
$\mathrm{~K}^{+}-p$-nitrophenol- (b) & -- & -3.3 \\
\hline
\end{tabular}

Bond lengths are in $\AA$; frequencies are in $\mathrm{cm}^{-1}$; bond angles are in degrees. ${ }^{a} \mathrm{~d}$ is the distance between metal ion and the negative oxygen atom. ${ }^{\mathrm{b}} \Delta \theta=\theta_{\text {complex }}-\theta_{\text {monomer, }}$, is the change in the ONO angle as compared with the $p$-nitrophenol monomer in the deprotonated form. 
Table S3. Cartesian coordinates, energies, vertical excitation energies and oscillator strengths of optimized geometries of studied $p$-nitrophenol-containing complexes obtained from DFT calculations.

\section{p-nitrophenol}

- Cartesian coordinates:

\begin{tabular}{lccc}
\hline \multirow{2}{*}{ tomic number } & \multicolumn{3}{c}{ Coordinates (Angstroms) } \\
\cline { 2 - 4 } & $\mathrm{X}$ & $\mathrm{Y}$ & $\mathrm{Z}$ \\
\hline 6 & 1.381440 & -1.199218 & 0.000000 \\
6 & -0.002460 & -1.207384 & 0.000000 \\
6 & -0.687689 & 0.000585 & 0.000000 \\
6 & -0.010577 & 1.216324 & 0.000000 \\
6 & 1.370869 & 1.223279 & 0.000000 \\
6 & 2.071908 & 0.015212 & 0.000000 \\
1 & 1.928787 & -2.134645 & 0.000001 \\
1 & -0.556190 & -2.134238 & 0.000001 \\
1 & -0.573423 & 2.137682 & -0.000001 \\
1 & 1.925978 & 2.151055 & -0.000001 \\
7 & -2.154262 & -0.006838 & 0.000000 \\
8 & -2.719940 & -1.091748 & -0.000001 \\
8 & -2.730311 & 1.072146 & 0.000001 \\
8 & 3.425698 & 0.086278 & 0.000000 \\
1 & 3.810163 & -0.798183 & 0.000000
\end{tabular}

- Energies (a.u.):

Zero-point correction $=$

Thermal correction to Energy=

0.107207 (Hartree/Particle)
0.115132
0.116076
0.073942
-512.087619
-512.079694
-512.078750

Thermal correction to Enthalpy=

Thermal correction to Gibbs Free Energy=

Sum of electronic and zero-point Energies=

Sum of electronic and thermal Energies=

$-512.078750$ 
- Vertical excitation energies and oscillator strengths (gas phase)

$\begin{array}{llllll}\text { Excited State } & \text { 1: } & \text { Singlet-A } & 3.8744 \mathrm{eV} & 320.01 \mathrm{~nm} & \mathrm{f}=0.0000 \\ \text { Excited State } & \text { 2: } & \text { Singlet-A } & 4.3480 \mathrm{eV} & 285.15 \mathrm{~nm} & \mathrm{f}=0.2585 \\ \text { Excited State } & \text { 3: } & \text { Singlet-A } & 4.4108 \mathrm{eV} & 281.09 \mathrm{~nm} & \mathrm{f}=0.0002 \\ \text { Excited State } & \text { 4: } & \text { Singlet-A } & 4.6177 \mathrm{eV} & 268.50 \mathrm{~nm} & \mathrm{f}=0.0041\end{array}$

- Vertical excitation energies and oscillator strengths (aqueous phase)

$\begin{array}{llllll}\text { Excited State } & \text { 1: } & \text { Singlet-A } & 3.9955 \mathrm{eV} & 310.31 \mathrm{~nm} & \mathrm{f}=0.3302 \\ \text { Excited State } & \text { 2: } & \text { Singlet-A } & 4.0146 \mathrm{eV} & 308.83 \mathrm{~nm} & \mathrm{f}=0.0000 \\ \text { Excited State } & \text { 3: } & \text { Singlet-A } & 4.2941 \mathrm{eV} & 288.73 \mathrm{~nm} & \mathrm{f}=0.0083 \\ \text { Excited State } & \text { 4: } & \text { Singlet-A } & 4.5410 \mathrm{eV} & 273.03 \mathrm{~nm} & \mathrm{f}=0.0003 \\ \text { Excited State } & \text { 5: } & \text { Singlet-A } & 5.3798 \mathrm{eV} & 230.46 \mathrm{~nm} & \mathrm{f}=0.0619 \\ \text { Excited State } & \text { 6: } & \text { Singlet-A } & 6.3121 \mathrm{eV} & 196.42 \mathrm{~nm} & \mathrm{f}=0.0965 \\ \text { Excited State } & 7: & \text { Singlet-A } & 6.3246 \mathrm{eV} & 196.04 \mathrm{~nm} & \mathrm{f}=0.0787 \\ \text { Excited State } & 8: & \text { Singlet-A } & 6.3345 \mathrm{eV} & 195.73 \mathrm{~nm} & \mathrm{f}=0.0000 \\ \text { Excited State } & 9: & \text { Singlet-A } & 6.6095 \mathrm{eV} & 187.58 \mathrm{~nm} & \mathrm{f}=0.0748 \\ \text { Excited State } & 10: & \text { Singlet-A } & 6.6228 \mathrm{eV} & 187.21 \mathrm{~nm} & \mathrm{f}=0.0000\end{array}$

\section{$\underline{M^{2+}-p-n i t r o p h e n o l}$}

$\mathrm{Mg}^{2+}-p$-nitrophenol (a)

- Cartesian coordinates:

\begin{tabular}{lccc}
\hline \multirow{2}{*}{ Atomic number } & \multicolumn{3}{c}{ Coordinates (Angstroms) } \\
\cline { 2 - 4 } & $\mathrm{X}$ & $\mathrm{Y}$ & $\mathrm{Z}$ \\
\hline 6 & 1.301967 & 1.238644 & -0.195144 \\
6 & -0.106691 & 1.231620 & -0.172401 \\
6 & -0.800622 & 0.013253 & -0.210583 \\
6 & -0.104890 & -1.208647 & -0.274346 \\
6 & 1.299254 & -1.211018 & -0.304435 \\
6 & 2.022342 & 0.020050 & -0.378895 \\
1 & 1.829416 & 2.186181 & -0.180859 \\
1 & -0.666337 & 2.159763 & -0.115070 \\
1 & -0.664417 & -2.138408 & -0.293233
\end{tabular}




$\begin{array}{rrrr}1 & 1.849608 & -2.142058 & -0.380804 \\ 7 & -2.311539 & 0.013790 & -0.217619 \\ 8 & -2.829442 & 1.104841 & -0.219186 \\ 8 & -2.828931 & -1.078278 & -0.209223 \\ 8 & 3.315426 & -0.069874 & -0.553452 \\ 1 & 3.785578 & 0.772198 & -0.684545 \\ 12 & 0.593528 & -0.090927 & 1.687296\end{array}$

- Energies (a.u.):

Zero-point correction $=$

Thermal correction to Energy=

0.108169 (Hartree/Particle)
0.117663
0.118607
0.071964
-711.489549
-711.480055
-711.479111
-711.525754

Thermal correction to Enthalpy=

Thermal correction to Gibbs Free Energy=

Sum of electronic and zero-point Energies=

Sum of electronic and thermal Energies=

Sum of electronic and thermal Enthalpies=

Sum of electronic and thermal Free Energies=

$-711.525754$

- Vertical excitation energies and oscillator strengths (gas phase)

$\begin{array}{lcllll}\text { Excited State } & 1: & \text { Singlet-A } & 2.2113 \mathrm{eV} & 560.69 \mathrm{~nm} & \mathrm{f}=0.0000 \\ \text { Excited State } & 2: & \text { Singlet-A } & 2.4053 \mathrm{eV} & 515.45 \mathrm{~nm} & \mathrm{f}=0.0005 \\ \text { Excited State } & 3: & \text { Singlet-A } & 2.5314 \mathrm{eV} & 489.79 \mathrm{~nm} & \mathrm{f}=0.0001 \\ \text { Excited State } & 4: & \text { Singlet-A } & 2.8462 \mathrm{eV} & 435.61 \mathrm{~nm} & \mathrm{f}=0.0078 \\ \text { Excited State } & 5: & \text { Singlet-A } & 3.6134 \mathrm{eV} & 343.13 \mathrm{~nm} & \mathrm{f}=0.0001 \\ \text { Excited State } & 6: & \text { Singlet-A } & 3.8772 \mathrm{eV} & 319.78 \mathrm{~nm} & \mathrm{f}=0.0005 \\ \text { Excited State } & 7: & \text { Singlet-A } & 4.0121 \mathrm{eV} & 309.02 \mathrm{~nm} & \mathrm{f}=0.0018 \\ \text { Excited State } & 8: & \text { Singlet-A } & 4.0214 \mathrm{eV} & 308.31 \mathrm{~nm} & \mathrm{f}=0.0008 \\ \text { Excited State } & 9: & \text { Singlet-A } & 4.1674 \mathrm{eV} & 297.51 \mathrm{~nm} & \mathrm{f}=0.0001 \\ \text { Excited State } & 10: & \text { Singlet-A } & 4.3873 \mathrm{eV} & 282.60 \mathrm{~nm} & \mathrm{f}=0.0054 \\ \text { Excited State } & 11: & \text { Singlet-A } & 4.7328 \mathrm{eV} & 261.97 \mathrm{~nm} & \mathrm{f}=0.0100 \\ \text { Excited State } & 12: & \text { Singlet-A } & 4.9091 \mathrm{eV} & 252.56 \mathrm{~nm} & \mathrm{f}=0.0261 \\ \text { Excited State } & 13: & \text { Singlet-A } & 5.0709 \mathrm{eV} & 244.50 \mathrm{~nm} & \mathrm{f}=0.0004 \\ \text { Excited State } & 14: & \text { Singlet-A } & 5.3903 \mathrm{eV} & 230.01 \mathrm{~nm} & \mathrm{f}=0.2262\end{array}$

$\mathrm{Mg}^{2+}-p$-nitrophenol (b)

- Cartesian coordinates:

\begin{tabular}{cc}
\hline Atomic number & Coordinates (Angstroms) \\
\hline
\end{tabular}




\begin{tabular}{cccc}
\hline & $\mathrm{X}$ & $\mathrm{Y}$ & $\mathrm{Z}$ \\
\hline 6 & 1.990606 & -1.241117 & 0.000001 \\
6 & 0.635695 & -1.248413 & 0.000000 \\
6 & -0.069334 & 0.002406 & 0.000000 \\
6 & 0.631609 & 1.255718 & 0.000001 \\
6 & 1.984966 & 1.250458 & 0.000000 \\
6 & 2.690336 & 0.006411 & 0.000000 \\
1 & 2.548477 & -2.169635 & 0.000002 \\
1 & 0.080831 & -2.175836 & -0.000002 \\
1 & 0.074223 & 2.181437 & 0.000000 \\
1 & 2.561494 & 2.166046 & 0.000001 \\
7 & -1.406065 & -0.001903 & 0.000000 \\
8 & -2.120522 & -1.095074 & -0.000000 \\
8 & -2.123545 & 1.088411 & -0.000000 \\
8 & 3.984952 & 0.091083 & -0.000002 \\
1 & 4.456652 & -0.761100 & 0.000002 \\
12 & -3.749131 & -0.004644 & -0.000000 \\
\hline & & &
\end{tabular}

- Energies (a.u.):

Zero-point correction=

0.109447 (Hartree/Particle)

Thermal correction to Energy=

0.118536

Thermal correction to Enthalpy=

0.119480

Thermal correction to Gibbs Free Energy=

Sum of electronic and zero-point Energies=

0.074670

Sum of electronic and thermal Energies $=$

$-711.589926$

Sum of electronic and thermal Enthalpies=

$-711.580838$

Sum of electronic and thermal Free Energies=

$-711.579893$

$-711.624703$

- Vertical excitation energies and oscillator strengths (gas phase)

$\begin{array}{llllll}\text { Excited State } & \text { 1: } & \text { Singlet-A } & 2.5151 \mathrm{eV} & 492.96 \mathrm{~nm} & \mathrm{f}=0.0025 \\ \text { Excited State } & \text { 2: } & \text { Singlet-A } & 3.0112 \mathrm{eV} & 411.75 \mathrm{~nm} & \mathrm{f}=0.0062 \\ \text { Excited State } & \text { 3: } & \text { Singlet-A } & 3.1443 \mathrm{eV} & 394.32 \mathrm{~nm} & \mathrm{f}=0.0000 \\ \text { Excited State } & \text { 4: } & \text { Singlet-A } & 3.7742 \mathrm{eV} & 328.51 \mathrm{~nm} & \mathrm{f}=0.6369 \\ \text { Excited State } & \text { 5: } & \text { Singlet-A } & 4.3106 \mathrm{eV} & 287.62 \mathrm{~nm} & \mathrm{f}=0.0000\end{array}$


$\mathrm{Mg}^{2+}-p$-nitrophenol (c)

- Cartesian coordinates:

\begin{tabular}{|c|c|c|c|}
\hline \multirow[t]{2}{*}{ Atomic number } & \multicolumn{3}{|c|}{ Coordinates (Angstroms) } \\
\hline & $X$ & $\mathrm{Y}$ & Z \\
\hline 6 & 1.469015 & -1.007409 & -0.551425 \\
\hline 6 & 0.093140 & -0.780847 & -0.626444 \\
\hline 6 & -0.425331 & 0.465655 & -0.167358 \\
\hline 6 & 0.412268 & 1.462238 & 0.317707 \\
\hline 6 & 1.771205 & 1.214610 & 0.404196 \\
\hline 6 & 2.318435 & -0.020484 & -0.000534 \\
\hline 1 & 1.891404 & -1.901299 & -0.995216 \\
\hline 1 & -0.462452 & -1.343164 & -1.388607 \\
\hline 1 & -0.000843 & 2.407978 & 0.647092 \\
\hline 1 & 2.444494 & 1.972001 & 0.787255 \\
\hline 7 & -1.854207 & 0.678399 & -0.167696 \\
\hline 8 & -2.581738 & -0.390968 & 0.027868 \\
\hline 8 & -2.345172 & 1.744071 & -0.293400 \\
\hline 8 & 3.621073 & -0.152841 & 0.125760 \\
\hline 1 & 3.988601 & -0.990305 & -0.197853 \\
\hline 12 & -1.522288 & -1.874890 & 0.598544 \\
\hline
\end{tabular}

- Energies (a.u.):

Zero-point correction=

Thermal correction to Energy=

0.108134 (Hartree/Particle)

Thermal correction to Enthalpy=

0.117423

Thermal correction to Gibbs Free Energy=

0.118367

Sum of electronic and zero-point Energies=

0.073232

Sum of electronic and thermal Energies=

$-711.534101$

Sum of electronic and thermal Enthalpies=

$-711.524812$

$-711.523868$

Sum of electronic and thermal Free Energies=

$-711.569003$ 
- Vertical excitation energies and oscillator strengths (gas phase)

$\begin{array}{llllll}\text { Excited State } & \text { 1: } & \text { Singlet-A } & 2.0102 \mathrm{eV} & 616.77 \mathrm{~nm} & \mathrm{f}=0.0095 \\ \text { Excited State } & \text { 2: } & \text { Singlet-A } & 3.2308 \mathrm{eV} & 383.76 \mathrm{~nm} & \mathrm{f}=0.0570 \\ \text { Excited State } & \text { 3: } & \text { Singlet-A } & 3.5431 \mathrm{eV} & 349.93 \mathrm{~nm} & \mathrm{f}=0.1418 \\ \text { Excited State } & \text { 4: } & \text { Singlet-A } & 3.8159 \mathrm{eV} & 324.92 \mathrm{~nm} & \mathrm{f}=0.0999 \\ \text { Excited State } & \text { 5: } & \text { Singlet-A } & 4.2739 \mathrm{eV} & 290.10 \mathrm{~nm} & \mathrm{f}=0.0104\end{array}$

$\mathrm{Mg}^{2+}-p$-nitrophenol (d)

- Cartesian coordinates:

\begin{tabular}{lrrr}
\hline \multirow{2}{*}{ Atomic number } & \multicolumn{3}{c}{ Coordinates (Angstroms) } \\
\cline { 2 - 4 } & $\mathrm{X}$ & $\mathrm{Y}$ & $\mathrm{Z}$ \\
\hline 6 & -0.685182 & 1.637995 & -0.003251 \\
6 & 0.685808 & 1.358844 & 0.090382 \\
6 & 1.156933 & 0.057546 & -0.066680 \\
6 & 0.328165 & -0.994815 & -0.389251 \\
6 & -1.053652 & -0.734249 & -0.533400 \\
6 & -1.514742 & 0.590995 & -0.309896 \\
1 & -1.061527 & 2.635679 & 0.187425 \\
1 & 1.392375 & 2.147470 & 0.323090 \\
1 & 0.747213 & -1.973297 & -0.590209 \\
1 & -1.602865 & -1.369525 & -1.244193 \\
7 & 2.630052 & -0.206497 & 0.085238 \\
8 & 3.315695 & 0.755734 & 0.342043 \\
8 & 2.975144 & -1.358361 & -0.058053 \\
8 & -2.933703 & 0.645509 & -0.238565 \\
1 & -3.350791 & 1.500148 & -0.447954 \\
12 & -2.907986 & -1.111330 & 0.673696
\end{tabular}

- Energies (a.u.):

Zero-point correction=

0.106953 (Hartree/Particle)

Thermal correction to Energy=

0.116578

Thermal correction to Enthalpy=

0.117522 
Thermal correction to Gibbs Free Energy=

Sum of electronic and zero-point Energies=

Sum of electronic and thermal Energies $=$

Sum of electronic and thermal Enthalpies=

Sum of electronic and thermal Free Energies=
0.071122

$-711.494830$

$-711.485205$

$-711.484261$

$-711.530662$

- Vertical excitation energies and oscillator strengths (gas phase)

$\begin{array}{llllll}\text { Excited State } & \text { 1: } & \text { Singlet-A } & 1.2520 \mathrm{eV} & 990.27 \mathrm{~nm} & \mathrm{f}=0.0002 \\ \text { Excited State } & \text { 2: } & \text { Singlet-A } & 1.5020 \mathrm{eV} & 825.44 \mathrm{~nm} & \mathrm{f}=0.0032 \\ \text { Excited State } & \text { 3: } & \text { Singlet-A } & 1.6118 \mathrm{eV} & 769.24 \mathrm{~nm} & \mathrm{f}=0.0080 \\ \text { Excited State } & \text { 4: } & \text { Singlet-A } & 2.3214 \mathrm{eV} & 534.10 \mathrm{~nm} & \mathrm{f}=0.0018 \\ \text { Excited State } & \text { 5: } & \text { Singlet-A } & 3.2282 \mathrm{eV} & 384.07 \mathrm{~nm} & \mathrm{f}=0.1522\end{array}$

\section{$\underline{C a^{2+}-p-n i t r o p h e n o l}$}

$\mathrm{Ca}^{2+}-p$-nitrophenol (b)

- Cartesian coordinates:

\begin{tabular}{|c|c|c|c|}
\hline \multirow[t]{2}{*}{ Atomic number } & \multicolumn{3}{|c|}{ Coordinates (Angstroms) } \\
\hline & $X$ & $\mathrm{Y}$ & $\mathrm{Z}$ \\
\hline 6 & 2.386573 & -1.236039 & -0.000000 \\
\hline 6 & 1.028475 & -1.242587 & -0.000000 \\
\hline 6 & 0.325716 & 0.002071 & -0.000001 \\
\hline 6 & 1.024608 & 1.249784 & -0.000001 \\
\hline 6 & 2.380896 & 1.246034 & -0.000000 \\
\hline 6 & 3.085476 & 0.006726 & 0.000000 \\
\hline 1 & 2.942725 & -2.165347 & -0.000000 \\
\hline 1 & 0.475192 & -2.170554 & -0.000000 \\
\hline 1 & 0.468518 & 2.175888 & -0.000001 \\
\hline 1 & 2.955080 & 2.162716 & -0.000000 \\
\hline 7 & -1.022500 & -0.001940 & -0.000000 \\
\hline 8 & -1.718578 & -1.088680 & -0.000000 \\
\hline 8 & -1.720983 & 1.082628 & -0.000000 \\
\hline 8 & 4.385824 & 0.090495 & 0.000001 \\
\hline 1 & 4.849062 & -0.764748 & -0.000000 \\
\hline
\end{tabular}


- Energies (a.u.):

Zero-point correction $=$

Thermal correction to Energy=

0.108901 (Hartree/Particle)

Thermal correction to Enthalpy=

0.118315

Thermal correction to Gibbs Free Energy=

0.119259

Sum of electronic and zero-point Energies=

0.073186

Sum of electronic and thermal Energies=

$-1189.185737$

Sum of electronic and thermal Enthalpies=

$-1189.176324$

Sum of electronic and thermal Free Energies=

$-1189.175379$

$-1189.221452$

- Vertical excitation energies and oscillator strengths (gas phase)

$\begin{array}{llllll}\text { Excited State } & \text { 1: } & \text { Singlet-A } & 3.0741 \mathrm{eV} & 403.32 \mathrm{~nm} & \mathrm{f}=0.0074 \\ \text { Excited State } & \text { 2: } & \text { Singlet-A } & 3.6981 \mathrm{eV} & 335.26 \mathrm{~nm} & \mathrm{f}=0.6331 \\ \text { Excited State } & \text { 3: } & \text { Singlet-A } & 3.8797 \mathrm{eV} & 319.57 \mathrm{~nm} & \mathrm{f}=0.0031 \\ \text { Excited State } & \text { 4: } & \text { Singlet-A } & 4.1899 \mathrm{eV} & 295.91 \mathrm{~nm} & \mathrm{f}=0.0000 \\ \text { Excited State } & \text { 5: } & \text { Singlet-A } & 4.5757 \mathrm{eV} & 270.96 \mathrm{~nm} & \mathrm{f}=0.0000\end{array}$

$\mathrm{Ca}^{2+}-p$-nitrophenol (d)

- Cartesian coordinates:

\begin{tabular}{crrr}
\hline \multirow{2}{*}{ Atomic number } & \multicolumn{3}{c}{ Coordinates (Angstroms) } \\
\cline { 2 - 4 } & $\mathrm{X}$ & $\mathrm{Y}$ & $\mathrm{Z}$ \\
\hline 6 & 0.333617 & 1.667776 & -0.103381 \\
6 & -1.006537 & 1.332295 & -0.287014 \\
6 & -1.458242 & 0.074360 & 0.094000 \\
6 & -0.638385 & -0.860685 & 0.701719 \\
6 & 0.700435 & -0.523803 & 0.928356 \\
6 & 1.154399 & 0.735360 & 0.511127 \\
1 & 0.713074 & 2.632326 & -0.417841 \\
1 & -1.699838 & 2.031493 & -0.737236 \\
1 & -1.050488 & -1.806313 & 1.029078 \\
1 & 1.304338 & -1.133687 & 1.601739 \\
7 & -2.896942 & -0.281936 & -0.144929
\end{tabular}




$\begin{array}{rrrr}8 & -3.596456 & 0.596262 & -0.596616 \\ 8 & -3.214445 & -1.419190 & 0.131082 \\ 8 & 2.560453 & 0.922408 & 0.618338 \\ 1 & 2.833311 & 1.784942 & 0.975903 \\ 20 & 2.883503 & -0.844143 & -0.686421\end{array}$

- Energies (a.u.):

Zero-point correction=

Thermal correction to Energy=

0.107001 (Hartree/Particle)
0.116994
0.117938
0.069520
-1189.100343
-1189.090350
-1189.089406
-1189.137824

Thermal correction to Enthalpy=

Thermal correction to Gibbs Free Energy=

Sum of electronic and zero-point Energies=

Sum of electronic and thermal Energies=

Sum of electronic and thermal Enthalpies=

Sum of electronic and thermal Free Energies=

$-1189.137824$

- Vertical excitation energies and oscillator strengths (gas phase)

$\begin{array}{lcllll}\text { Excited State } & 1: & \text { Singlet-A } & 2.6596 \mathrm{eV} & 466.17 \mathrm{~nm} & \mathrm{f}=0.0000 \\ \text { Excited State } & 2: & \text { Singlet-A } & 2.9330 \mathrm{eV} & 422.72 \mathrm{~nm} & \mathrm{f}=0.0005 \\ \text { Excited State } & 3: & \text { Singlet-A } & 3.0205 \mathrm{eV} & 410.47 \mathrm{~nm} & \mathrm{f}=0.0005 \\ \text { Excited State } & 4: & \text { Singlet-A } & 3.0265 \mathrm{eV} & 409.67 \mathrm{~nm} & \mathrm{f}=0.0007 \\ \text { Excited State } & 5: & \text { Singlet-A } & 3.2966 \mathrm{eV} & 376.10 \mathrm{~nm} & \mathrm{f}=0.0000 \\ \text { Excited State } & 6: & \text { Singlet-A } & 3.2987 \mathrm{eV} & 375.86 \mathrm{~nm} & \mathrm{f}=0.0002 \\ \text { Excited State } & 7: & \text { Singlet-A } & 3.3402 \mathrm{eV} & 371.19 \mathrm{~nm} & \mathrm{f}=0.0000 \\ \text { Excited State } & 8: & \text { Singlet-A } & 3.4520 \mathrm{eV} & 359.16 \mathrm{~nm} & \mathrm{f}=0.0027 \\ \text { Excited State } & 9: & \text { Singlet-A } & 3.5172 \mathrm{eV} & 352.51 \mathrm{~nm} & \mathrm{f}=0.0000 \\ \text { Excited State } & 10: & \text { Singlet-A } & 3.5704 \mathrm{eV} & 347.26 \mathrm{~nm} & \mathrm{f}=0.0006\end{array}$

\section{$\underline{N a^{+}-p \text {-nitrophenol }}$}

$\mathrm{Na}^{+}-p$-nitrophenol (b)

- Cartesian coordinates:

\begin{tabular}{cccc}
\hline \multirow{2}{*}{ Atomic number } & \multicolumn{3}{c}{ Coordinates (Angstroms) } \\
\cline { 2 - 4 } & $\mathrm{X}$ & $\mathrm{Y}$ & $\mathrm{Z}$ \\
\hline 6 & 2.009944 & -1.212837 & -0.000000 \\
6 & 0.636014 & -1.219722 & 0.000001
\end{tabular}




$\begin{array}{lrrr}6 & -0.052752 & 0.001572 & -0.000000 \\ 6 & 0.631646 & 1.228253 & 0.000000 \\ 6 & 2.002999 & 1.229248 & 0.000000 \\ 6 & 2.704136 & 0.010184 & 0.000001 \\ 1 & 2.558503 & -2.146461 & -0.000003 \\ 1 & 0.086024 & -2.148768 & -0.000001 \\ 1 & 0.076368 & 2.154083 & -0.000001 \\ 1 & 2.565291 & 2.152319 & -0.000001 \\ 7 & -1.464984 & -0.002927 & 0.000000 \\ 8 & -2.087781 & -1.082871 & 0.000000 \\ 8 & -2.092440 & 1.073892 & -0.000000 \\ 8 & 4.035230 & 0.087248 & -0.000000 \\ 1 & 4.452776 & -0.784910 & -0.000002 \\ 11 & -4.174187 & -0.004738 & -0.000000\end{array}$

- Energies (a.u.):

Zero-point correction $=$

0.108607 (Hartree/Particle)
0.118375
0.119319
0.072507
-674.244192
-674.234424
-674.233480
-674.280293

Thermal correction to Energy=

Thermal correction to Enthalpy=

Thermal correction to Gibbs Free Energy=

Sum of electronic and zero-point Energies=

Sum of electronic and thermal Energies $=$

Sum of electronic and thermal Enthalpies=

Sum of electronic and thermal Free Energies=

- Vertical excitation energies and oscillator strengths (gas phase) 
$\mathrm{Na}^{+}-p$-nitrophenol (d)

- Cartesian coordinates:

\begin{tabular}{cccc}
\hline \multirow{2}{*}{ Atomic number } & \multicolumn{3}{c}{ Coordinates (Angstroms) } \\
\cline { 2 - 4 } & $\mathrm{X}$ & $\mathrm{Y}$ & $\mathrm{Z}$ \\
\hline 6 & -0.579810 & 1.501777 & -0.040477 \\
6 & 0.782891 & 1.292724 & 0.115730 \\
6 & 1.290070 & 0.010596 & -0.037006 \\
6 & 0.481281 & -1.066837 & -0.361546 \\
6 & -0.879737 & -0.854316 & -0.535618 \\
6 & -1.392078 & 0.423864 & -0.362789 \\
1 & -0.999733 & 2.491075 & 0.092987 \\
1 & 1.449785 & 2.105824 & 0.362374 \\
1 & 0.919822 & -2.045054 & -0.493032 \\
1 & -1.521362 & -1.666844 & -0.855310 \\
7 & 2.745508 & -0.214294 & 0.145995 \\
8 & 3.428086 & 0.758789 & 0.406702 \\
8 & -2.148731 & -1.356740 & 0.024850 \\
8 & -2.782199 & 0.581989 & -0.483606 \\
11 & -059629 & -0.777897 & 0.769911
\end{tabular}

- Energies (a.u.):

Zero-point correction=

Thermal correction to Energy=

0.107628 (Hartree/Particle)

Thermal correction to Enthalpy=

0.117841

Thermal correction to Gibbs Free Energy=

0.118785

Sum of electronic and zero-point Energies=

0.069369

Sum of electronic and thermal Energies=

$-674.206180$

$-674.195967$

Sum of electronic and thermal Enthalpies=

$-674.195023$

Sum of electronic and thermal Free Energies=

$-674.244439$ 
- Vertical excitation energies and oscillator strengths (gas phase)

$\begin{array}{llllll}\text { Excited State } & \text { 1: } & \text { Singlet-A } & 3.8074 \mathrm{eV} & 325.64 \mathrm{~nm} & \mathrm{f}=0.0001 \\ \text { Excited State } & \text { 2: } & \text { Singlet-A } & 4.2520 \mathrm{eV} & 291.59 \mathrm{~nm} & \mathrm{f}=0.0002 \\ \text { Excited State } & \text { 3: } & \text { Singlet-A } & 4.3203 \mathrm{eV} & 286.98 \mathrm{~nm} & \mathrm{f}=0.0016 \\ \text { Excited State } & \text { 4: } & \text { Singlet-A } & 4.5247 \mathrm{eV} & 274.02 \mathrm{~nm} & \mathrm{f}=0.0001 \\ \text { Excited State } & \text { 5: } & \text { Singlet-A } & 4.6234 \mathrm{eV} & 268.17 \mathrm{~nm} & \mathrm{f}=0.0073 \\ \text { Excited State } & \text { 6: } & \text { Singlet-A } & 4.8942 \mathrm{eV} & 253.33 \mathrm{~nm} & \mathrm{f}=0.0001 \\ \text { Excited State } & 7: & \text { Singlet-A } & 4.9333 \mathrm{eV} & 251.32 \mathrm{~nm} & \mathrm{f}=0.0035 \\ \text { Excited State } & 8: & \text { Singlet-A } & 5.0338 \mathrm{eV} & 246.30 \mathrm{~nm} & \mathrm{f}=0.0083 \\ \text { Excited State } & 9: & \text { Singlet-A } & 5.0793 \mathrm{eV} & 244.10 \mathrm{~nm} & \mathrm{f}=0.1996 \\ \text { Excited State } & 10: & \text { Singlet-A } & 5.4610 \mathrm{eV} & 227.04 \mathrm{~nm} & \mathrm{f}=0.0003\end{array}$

\section{$\underline{K^{+}-p \text {-nitrophenol }}$}

$\mathrm{K}^{+}-p$-nitrophenol (b)

- Cartesian coordinates:

\begin{tabular}{|c|c|c|c|}
\hline \multirow[t]{2}{*}{ Atomic number } & \multicolumn{3}{|c|}{ Coordinates (Angstroms) } \\
\hline & $X$ & $\mathrm{Y}$ & $\mathrm{Z}$ \\
\hline 6 & 2.480634 & -1.209887 & 0.000000 \\
\hline 6 & 1.104482 & -1.217194 & -0.000000 \\
\hline 6 & 0.416058 & 0.000696 & -0.000001 \\
\hline 6 & 1.098761 & 1.224885 & -0.000001 \\
\hline 6 & 2.472209 & 1.227457 & -0.000000 \\
\hline 6 & 3.174096 & 0.011171 & 0.000000 \\
\hline 1 & 3.028754 & -2.143878 & 0.000001 \\
\hline 1 & 0.555277 & -2.146603 & -0.000000 \\
\hline 1 & 0.543065 & 2.150364 & -0.000001 \\
\hline 1 & 3.032590 & 2.151709 & -0.000000 \\
\hline 7 & -1.007892 & -0.004523 & -0.000001 \\
\hline 8 & -1.617226 & -1.085619 & -0.000002 \\
\hline 8 & -1.622912 & 1.073113 & -0.000000 \\
\hline 8 & 4.509044 & 0.088371 & 0.000001 \\
\hline 1 & 4.922219 & -0.785283 & 0.000003 \\
\hline 19 & -4.192387 & -0.001281 & 0.000001 \\
\hline
\end{tabular}


- Energies (a.u.):

Zero-point correction=

Thermal correction to Energy=

0.108152 (Hartree/Particle)

Thermal correction to Enthalpy=

0.118231

Thermal correction to Gibbs Free Energy=

Sum of electronic and zero-point Energies=

0.119175

Sum of electronic and thermal Energies=

0.070523

$-1111.896787$

$-1111.886707$

$-1111.885763$

Sum of electronic and thermal Enthalpies=

$-1111.934416$

Sum of electronic and thermal Free Energies=

- Vertical excitation energies and oscillator strengths (gas phase)

$\begin{array}{lcllll}\text { Excited State } & 1: & \text { Singlet-A } & 3.7275 \mathrm{eV} & 332.62 \mathrm{~nm} & \mathrm{f}=0.3183 \\ \text { Excited State } & 2: & \text { Singlet-A } & 3.8165 \mathrm{eV} & 324.87 \mathrm{~nm} & \mathrm{f}=0.0739 \\ \text { Excited State } & 3: & \text { Singlet-A } & 3.9129 \mathrm{eV} & 316.86 \mathrm{~nm} & \mathrm{f}=0.0000 \\ \text { Excited State } & 4: & \text { Singlet-A } & 4.7995 \mathrm{eV} & 258.32 \mathrm{~nm} & \mathrm{f}=0.0006 \\ \text { Excited State } & \text { 5: } & \text { Singlet-A } & 5.2315 \mathrm{eV} & 237.00 \mathrm{~nm} & \mathrm{f}=0.0009 \\ \text { Excited State } & 6: & \text { Singlet-A } & 5.4014 \mathrm{eV} & 229.54 \mathrm{~nm} & \mathrm{f}=0.0293 \\ \text { Excited State } & 7: & \text { Singlet-A } & 5.7445 \mathrm{eV} & 215.83 \mathrm{~nm} & \mathrm{f}=0.0000 \\ \text { Excited State } & 8: & \text { Singlet-A } & 6.0061 \mathrm{eV} & 206.43 \mathrm{~nm} & \mathrm{f}=0.0000 \\ \text { Excited State } & 9: & \text { Singlet-A } & 6.0285 \mathrm{eV} & 205.66 \mathrm{~nm} & \mathrm{f}=0.0812 \\ \text { Excited State } & 10: & \text { Singlet-A } & 6.1061 \mathrm{eV} & 203.05 \mathrm{~nm} & \mathrm{f}=0.0630\end{array}$

$\mathrm{K}^{+}-p$-nitrophenol (d)

- Cartesian coordinates:

\begin{tabular}{lrrr}
\hline \multirow{2}{*}{ tomic number } & \multicolumn{3}{c}{ Coordinates (Angstroms) } \\
\cline { 2 - 4 } & \multicolumn{1}{c}{$\mathrm{X}$} & \multicolumn{1}{c}{$\mathrm{Y}$} & \multicolumn{1}{c}{$\mathrm{Z}$} \\
\hline 6 & 0.057216 & 1.655972 & -0.079891 \\
6 & -1.261755 & 1.289236 & -0.302338 \\
6 & -1.675604 & 0.011822 & 0.047030 \\
6 & -0.814753 & -0.902409 & 0.634269 \\
6 & 0.500825 & -0.529351 & 0.871193 \\
6 & 0.927645 & 0.741555 & 0.502065 \\
1 & 0.402580 & 2.643685 & -0.360012 \\
1 & -1.966340 & 1.975712 & -0.748445 \\
1 & -1.182719 & -1.878099 & 0.915652 \\
& 16 & & \\
\hline
\end{tabular}




$\begin{array}{rrrr}1 & 1.173797 & -1.199381 & 1.392254 \\ 7 & -3.080220 & -0.385339 & -0.207476 \\ 8 & -3.815020 & 0.452516 & -0.697673 \\ 8 & -3.397874 & -1.523790 & 0.088145 \\ 8 & 2.272418 & 1.042663 & 0.696745 \\ 1 & 2.380279 & 1.991602 & 0.842975 \\ 19 & 3.888227 & -0.747800 & -0.595884\end{array}$

- Energies (a.u.):

Zero-point correction=

Thermal correction to Energy=

0.107472 (Hartree/Particle)
0.117837
0.118782

Thermal correction to Enthalpy= 0.068085

Sum of electronic and zero-point Energies=

$-1111.864134$

Sum of electronic and thermal Energies=

$-1111.853768$

Sum of electronic and thermal Enthalpies=

$-1111.852824$

Sum of electronic and thermal Free Energies=

$-1111.903521$

- Vertical excitation energies and oscillator strengths (gas phase)

$\begin{array}{lcllll}\text { Excited State } & 1: & \text { Singlet-A } & 3.8223 \mathrm{eV} & 324.37 \mathrm{~nm} & \mathrm{f}=0.0001 \\ \text { Excited State } & 2: & \text { Singlet-A } & 4.2770 \mathrm{eV} & 289.89 \mathrm{~nm} & \mathrm{f}=0.0002 \\ \text { Excited State } & 3: & \text { Singlet-A } & 4.7294 \mathrm{eV} & 262.16 \mathrm{~nm} & \mathrm{f}=0.0019 \\ \text { Excited State } & 4: & \text { Singlet-A } & 4.9118 \mathrm{eV} & 252.42 \mathrm{~nm} & \mathrm{f}=0.0048 \\ \text { Excited State } & 5: & \text { Singlet-A } & 4.9486 \mathrm{eV} & 250.55 \mathrm{~nm} & \mathrm{f}=0.2005 \\ \text { Excited State } & 6: & \text { Singlet-A } & 5.0748 \mathrm{eV} & 244.32 \mathrm{~nm} & \mathrm{f}=0.0008 \\ \text { Excited State } & 7: & \text { Singlet-A } & 5.1691 \mathrm{eV} & 239.85 \mathrm{~nm} & \mathrm{f}=0.0253 \\ \text { Excited State } & 8: & \text { Singlet-A } & 5.4535 \mathrm{eV} & 227.35 \mathrm{~nm} & \mathrm{f}=0.0015 \\ \text { Excited State } & 9: & \text { Singlet-A } & 5.5355 \mathrm{eV} & 223.98 \mathrm{~nm} & \mathrm{f}=0.0006 \\ \text { Excited State } & 10: & \text { Singlet-A } & 5.5873 \mathrm{eV} & 221.90 \mathrm{~nm} & \mathrm{f}=0.0013\end{array}$

\section{p-nitrophenol- $\mathrm{NO}_{3}^{-}$}

- Cartesian coordinates:

\begin{tabular}{|c|c|c|c|}
\hline \multirow{2}{*}{ Atomic number } & \multicolumn{3}{|c|}{ Coordinates (Angstroms) } \\
\hline & $\mathrm{X}$ & Y & Z \\
\hline 6 & -0.106793 & -0.270463 & -0.078758 \\
\hline 6 & 1.139105 & -0.858074 & -0.104795 \\
\hline
\end{tabular}




$\begin{array}{lrrr}6 & 2.290442 & -0.069730 & -0.018439 \\ 6 & 2.190040 & 1.318260 & 0.104065 \\ 6 & 0.947172 & 1.909289 & 0.130193 \\ 6 & -0.227685 & 1.132463 & 0.033120 \\ 1 & -1.008210 & -0.867105 & -0.102563 \\ 1 & 1.241176 & -1.930372 & -0.184487 \\ 1 & 3.091976 & 1.908194 & 0.173797 \\ 1 & 0.841701 & 2.982433 & 0.220470 \\ 7 & 3.594144 & -0.691160 & -0.050124 \\ 8 & 3.661801 & -1.915261 & -0.166000 \\ 8 & 4.592243 & 0.027616 & 0.039167 \\ 8 & -1.386698 & 1.757208 & 0.055689 \\ 1 & -2.199533 & 1.146833 & -0.137853 \\ 8 & -3.464646 & 0.482456 & -0.633489 \\ 8 & -3.118322 & -1.019471 & 0.907204 \\ 8 & -4.968269 & -1.027541 & -0.229648 \\ 7 & -3.864097 & -0.544625 & 0.029399\end{array}$

- Energies (a.u.):

Zero-point correction=

Thermal correction to Energy=

0.122136 (Hartree/Particle)

$$
0.134521
$$

Thermal correction to Enthalpy=

0.135465

Thermal correction to Gibbs Free Energy= 0.079959

Sum of electronic and zero-point Energies=

$-792.615201$

Sum of electronic and thermal Energies=

$-792.602816$

Sum of electronic and thermal Enthalpies=

$-792.601872$

Sum of electronic and thermal Free Energies=

$-792.657378$

- Vertical excitation energies and oscillator strengths (gas phase)

$\begin{array}{llllll}\text { Excited State } & \text { 1: } & \text { Singlet-A } & 2.9524 \mathrm{eV} & 419.95 \mathrm{~nm} & \mathrm{f}=0.0003 \\ \text { Excited State } & \text { 2: } & \text { Singlet-A } & 3.7940 \mathrm{eV} & 326.79 \mathrm{~nm} & \mathrm{f}=0.3441 \\ \text { Excited State } & \text { 3: } & \text { Singlet-A } & 3.8689 \mathrm{eV} & 320.46 \mathrm{~nm} & \mathrm{f}=0.0063 \\ \text { Excited State } & \text { 4: } & \text { Singlet-A } & 4.1746 \mathrm{eV} & 297.00 \mathrm{~nm} & \mathrm{f}=0.0004 \\ \text { Excited State } & \text { 5: } & \text { Singlet-A } & 4.1970 \mathrm{eV} & 295.41 \mathrm{~nm} & \mathrm{f}=0.0000\end{array}$




$\begin{array}{lrllll}\text { Excited State } & \text { 6: } & \text { Singlet-A } & 4.2767 \mathrm{eV} & 289.91 \mathrm{~nm} & \mathrm{f}=0.0019 \\ \text { Excited State } & \text { 7: } & \text { Singlet-A } & 4.3313 \mathrm{eV} & 286.25 \mathrm{~nm} & \mathrm{f}=0.0029 \\ \text { Excited State } & \text { 8: } & \text { Singlet-A } & 4.5042 \mathrm{eV} & 275.27 \mathrm{~nm} & \mathrm{f}=0.0003 \\ \text { Excited State } & \text { 9: } & \text { Singlet-A } & 4.5820 \mathrm{eV} & 270.59 \mathrm{~nm} & \mathrm{f}=0.0067 \\ \text { Excited State } & 10: & \text { Singlet-A } & 4.6073 \mathrm{eV} & 269.10 \mathrm{~nm} & \mathrm{f}=0.0004\end{array}$

\section{p-nitrophenol-Cl}

- Cartesian coordinates:

\begin{tabular}{cccc}
\hline \multirow{2}{*}{ Atomic number } & \multicolumn{3}{c}{ Coordinates (Angstroms) } \\
\cline { 2 - 4 } & $\mathrm{X}$ & $\mathrm{Y}$ & $\mathrm{Z}$ \\
\hline 6 & 0.699307 & 0.532925 & 0.000001 \\
6 & -0.611524 & 0.954777 & 0.000000 \\
6 & -1.653828 & 0.021474 & 0.000000 \\
6 & -1.375157 & -1.348148 & -0.000000 \\
6 & -0.067182 & -1.775589 & 0.000000 \\
6 & 1.000476 & -0.849752 & 0.000000 \\
1 & 1.523597 & 1.234806 & 0.000002 \\
1 & -0.851448 & 2.008026 & 0.000001 \\
1 & -2.194059 & -2.052371 & -0.000000 \\
1 & 0.174574 & -2.830413 & 0.000001 \\
7 & -3.024472 & 0.470828 & -0.000000 \\
8 & -3.249946 & 1.682456 & 0.000000 \\
8 & -3.923813 & -0.374236 & -0.000001 \\
8 & 2.228532 & -1.319309 & -0.000000 \\
17 & 2.948907 & -0.564762 & -0.000000 \\
\hline & 4.186999 & 0.810795 & -0.000000
\end{tabular}

- Energies (a.u.):

Zero-point correction=
0.106713 (Hartree/Particle)
0.116386
0.117330
0.069593
$-972.443072$

Thermal correction to Energy=

Thermal correction to Enthalpy=

Thermal correction to Gibbs Free Energy=

Sum of electronic and zero-point Energies= 
Sum of electronic and thermal Energies=

$-972.433399$

Sum of electronic and thermal Enthalpies=

$-972.432454$

Sum of electronic and thermal Free Energies=

$-972.480192$

- Vertical excitation energies and oscillator strengths (gas phase)

$\begin{array}{llllll}\text { Excited State } & \text { 1: } & \text { Singlet-A } & 2.5194 \mathrm{eV} & 492.12 \mathrm{~nm} & \mathrm{f}=0.0033 \\ \text { Excited State } & \text { 2: } & \text { Singlet-A } & 2.5398 \mathrm{eV} & 488.17 \mathrm{~nm} & \mathrm{f}=0.0000 \\ \text { Excited State } & \text { 3: } & \text { Singlet-A } & 2.8086 \mathrm{eV} & 441.45 \mathrm{~nm} & \mathrm{f}=0.0000 \\ \text { Excited State } & \text { 4: } & \text { Singlet-A } & 3.7684 \mathrm{eV} & 329.01 \mathrm{~nm} & \mathrm{f}=0.3551 \\ \text { Excited State } & \text { 5: } & \text { Singlet-A } & 3.8682 \mathrm{eV} & 320.52 \mathrm{~nm} & \mathrm{f}=0.0000 \\ \text { Excited State } & \text { 6: } & \text { Singlet-A } & 4.1577 \mathrm{eV} & 298.21 \mathrm{~nm} & \mathrm{f}=0.0085 \\ \text { Excited State } & 7: & \text { Singlet-A } & 4.1617 \mathrm{eV} & 297.91 \mathrm{~nm} & \mathrm{f}=0.0000 \\ \text { Excited State } & 8: & \text { Singlet-A } & 4.2977 \mathrm{eV} & 288.49 \mathrm{~nm} & \mathrm{f}=0.0037 \\ \text { Excited State } & \text { 9: } & \text { Singlet-A } & 4.4307 \mathrm{eV} & 279.83 \mathrm{~nm} & \mathrm{f}=0.0000 \\ \text { Excited State } & 10: & \text { Singlet-A } & 4.5255 \mathrm{eV} & 273.97 \mathrm{~nm} & \mathrm{f}=0.0003\end{array}$

\section{$\underline{\mathrm{Mg}^{2+}-\text { p-nitrophenol- } \mathrm{NO}_{3}^{-}} \underline{-}^{-}$}

- Cartesian coordinates:

\begin{tabular}{|c|c|c|c|}
\hline \multirow[t]{2}{*}{ Atomic number } & \multicolumn{3}{|c|}{ Coordinates (Angstroms) } \\
\hline & $\mathrm{X}$ & $\mathrm{Y}$ & $\mathrm{Z}$ \\
\hline 6 & -0.561226 & -0.249468 & -0.181004 \\
\hline 6 & 0.700649 & -0.703706 & -0.165998 \\
\hline 6 & 1.793597 & 0.234966 & -0.024360 \\
\hline 6 & 1.552346 & 1.657993 & 0.099164 \\
\hline 6 & 0.290186 & 2.109914 & 0.083633 \\
\hline 6 & -0.854942 & 1.189982 & -0.055334 \\
\hline 1 & -1.411309 & -0.911595 & -0.271421 \\
\hline 1 & 0.930687 & -1.755197 & -0.253856 \\
\hline 1 & 2.399646 & 2.319695 & 0.201382 \\
\hline 1 & 0.057230 & 3.162599 & 0.172907 \\
\hline 7 & 3.025720 & -0.210991 & -0.005478 \\
\hline 8 & 3.351341 & -1.512215 & -0.112520 \\
\hline 8 & 4.107508 & 0.579802 & 0.125041 \\
\hline 8 & -1.999162 & 1.622346 & -0.065292 \\
\hline
\end{tabular}




$\begin{array}{llll}1 & -3.619688 & 0.843430 & -0.125054 \\ 8 & -4.572283 & 0.587043 & -0.186913 \\ 8 & -3.542781 & -1.314624 & 0.287485 \\ 8 & -5.701709 & -1.252749 & 0.057393 \\ 7 & -4.612694 & -0.769258 & 0.073432 \\ 12 & 5.173775 & -0.992675 & 0.035185\end{array}$

- Energies (a.u.):

Zero-point correction=

Thermal correction to Energy=

0.124122 (Hartree/Particle)

Thermal correction to Enthalpy=

0.138451

Thermal correction to Gibbs Free Energy=

0.139395

Sum of electronic and zero-point Energies $=$

0.078236

Sum of electronic and thermal Energies=

$-992.357464$

$-992.343135$

Sum of electronic and thermal Enthalpies=

$-992.342190$

Sum of electronic and thermal Free Energies=

$-992.403350$

- Vertical excitation energies and oscillator strengths (gas phase)

$\begin{array}{llllll}\text { Excited State } & 1: & \text { Singlet-A } & 1.3771 \mathrm{eV} & 900.31 \mathrm{~nm} & \mathrm{f}=0.0020 \\ \text { Excited State } & \text { 2: } & \text { Singlet-A } & 1.4034 \mathrm{eV} & 883.47 \mathrm{~nm} & \mathrm{f}=0.0006 \\ \text { Excited State } & 3: & \text { Singlet-A } & 1.8159 \mathrm{eV} & 682.79 \mathrm{~nm} & \mathrm{f}=0.0000 \\ \text { Excited State } & \text { 4: } & \text { Singlet-A } & 2.0437 \mathrm{eV} & 606.65 \mathrm{~nm} & \mathrm{f}=0.0000 \\ \text { Excited State } & 5: & \text { Singlet-A } & 2.3917 \mathrm{eV} & 518.39 \mathrm{~nm} & \mathrm{f}=0.0000 \\ \text { Excited State } & \text { 6: } & \text { Singlet-A } & 2.4006 \mathrm{eV} & 516.48 \mathrm{~nm} & \mathrm{f}=0.0000 \\ \text { Excited State } & 7: & \text { Singlet-A } & 2.5121 \mathrm{eV} & 493.54 \mathrm{~nm} & \mathrm{f}=0.0000 \\ \text { Excited State } & 8: & \text { Singlet-A } & 2.6737 \mathrm{eV} & 463.72 \mathrm{~nm} & \mathrm{f}=0.0004 \\ \text { Excited State } & 9: & \text { Singlet-A } & 2.6934 \mathrm{eV} & 460.32 \mathrm{~nm} & \mathrm{f}=0.0000 \\ \text { Excited State } & 10: & \text { Singlet-A } & 3.3306 \mathrm{eV} & 372.26 \mathrm{~nm} & \mathrm{f}=0.0002 \\ \text { Excited State } & 11: & \text { Singlet-A } & 3.5788 \mathrm{eV} & 346.44 \mathrm{~nm} & \mathrm{f}=0.0063 \\ \text { Excited State } & 12: & \text { Singlet-A } & 3.6733 \mathrm{eV} & 337.53 \mathrm{~nm} & \mathrm{f}=0.0000 \\ \text { Excited State } & 13: & \text { Singlet-A } & 3.7653 \mathrm{eV} & 329.28 \mathrm{~nm} & \mathrm{f}=0.0010 \\ \text { Excited State } & 14: & \text { Singlet-A } & 3.8070 \mathrm{eV} & 325.67 \mathrm{~nm} & \mathrm{f}=0.0000 \\ \text { Excited State } & 15: & \text { Singlet-A } & 3.8922 \mathrm{eV} & 318.55 \mathrm{~nm} & \mathrm{f}=0.4113 \\ \text { Excited State } & 16: & \text { Singlet-A } & 3.9746 \mathrm{eV} & 311.94 \mathrm{~nm} & \mathrm{f}=0.3187\end{array}$

- Vertical excitation energies and oscillator strengths (aqueous phase)

$\begin{array}{llllll}\text { Excited State } & 1: & \text { Singlet-A } & 3.1232 \mathrm{eV} & 396.98 \mathrm{~nm} & \mathrm{f}=0.0000 \\ \text { Excited State } & \text { 2: } & \text { Singlet-A } & 3.7091 \mathrm{eV} & 334.27 \mathrm{~nm} & \mathrm{f}=0.7492 \\ \text { Excited State } & \text { 3: } & \text { Singlet-A } & 3.8591 \mathrm{eV} & 321.28 \mathrm{~nm} & \mathrm{f}=0.0012\end{array}$




$\begin{array}{lrllll}\text { Excited State } & \text { 4: } & \text { Singlet-A } & 4.3760 \mathrm{eV} & 283.33 \mathrm{~nm} & \mathrm{f}=0.0004 \\ \text { Excited State } & \text { 5: } & \text { Singlet-A } & 4.3832 \mathrm{eV} & 282.86 \mathrm{~nm} & \mathrm{f}=0.0000 \\ \text { Excited State } & \text { 6: } & \text { Singlet-A } & 4.7903 \mathrm{eV} & 258.83 \mathrm{~nm} & \mathrm{f}=0.0002 \\ \text { Excited State } & \text { 7: } & \text { Singlet-A } & 4.8815 \mathrm{eV} & 253.99 \mathrm{~nm} & \mathrm{f}=0.0001 \\ \text { Excited State } & \text { 8: } & \text { Singlet-A } & 4.8905 \mathrm{eV} & 253.52 \mathrm{~nm} & \mathrm{f}=0.0004 \\ \text { Excited State } & \text { 9: } & \text { Singlet-A } & 5.1505 \mathrm{eV} & 240.72 \mathrm{~nm} & \mathrm{f}=0.0283 \\ \text { Excited State } & 10: & \text { Singlet-A } & 5.3665 \mathrm{eV} & 231.03 \mathrm{~nm} & \mathrm{f}=0.0107 \\ \text { Excited State } & 11: & \text { Singlet-A } & 5.3924 \mathrm{eV} & 229.92 \mathrm{~nm} & \mathrm{f}=0.0484 \\ \text { Excited State } & 12: & \text { Singlet-A } & 5.5133 \mathrm{eV} & 224.88 \mathrm{~nm} & \mathrm{f}=0.0038 \\ \text { Excited State } & 13: & \text { Singlet-A } & 5.5684 \mathrm{eV} & 222.66 \mathrm{~nm} & \mathrm{f}=0.0000 \\ \text { Excited State } & 14: & \text { Singlet-A } & 5.7181 \mathrm{eV} & 216.83 \mathrm{~nm} & \mathrm{f}=0.0030 \\ \text { Excited State } & 15: & \text { Singlet-A } & 5.7987 \mathrm{eV} & 213.81 \mathrm{~nm} & \mathrm{f}=0.0003 \\ \text { Excited State } & 16: & \text { Singlet-A } & 5.8633 \mathrm{eV} & 211.46 \mathrm{~nm} & \mathrm{f}=0.0001\end{array}$

\section{$\underline{\mathrm{Mg}^{2+}-p \text {-nitrophenol-Cl}}$}

- Cartesian coordinates:

\begin{tabular}{cccc}
\hline \multirow{2}{*}{ Atomic number } & \multicolumn{3}{c}{ Coordinates (Angstroms) } \\
\cline { 2 - 4 } & $\mathrm{X}$ & $\mathrm{Y}$ & $\mathrm{Z}$ \\
\hline 6 & 1.256333 & -0.398016 & -0.000003 \\
6 & -0.028131 & -0.782750 & -0.000003 \\
6 & -1.071779 & 0.221146 & -0.000001 \\
6 & -0.756359 & 1.634991 & 0.000000 \\
6 & 0.528457 & 2.017392 & 0.000000 \\
6 & 1.629216 & 1.032080 & -0.000001 \\
1 & 2.066066 & -1.116031 & -0.000004 \\
1 & -0.314270 & -1.824110 & -0.000004 \\
1 & -1.569279 & 2.345891 & 0.000001 \\
1 & 0.815504 & 3.060508 & 0.000001 \\
7 & -2.326151 & -0.156535 & -0.000001 \\
8 & -2.720561 & -1.444805 & -0.000002 \\
8 & -3.367469 & 0.699083 & 0.000002 \\
8 & 2.793952 & 1.396630 & -0.000001 \\
1 & 4.264059 & 0.035481 & 0.000001
\end{tabular}


- Energies (a.u.):

Zero-point correction=

Thermal correction to Energy=

0.105152 (Hartree/Particle)
0.117230
0.118175

Thermal correction to Enthalpy=

0.064090

Thermal correction to Gibbs Free Energy=

$-1172.191228$

Sum of electronic and thermal Energies=

$-1172.179149$

Sum of electronic and thermal Enthalpies=

$-1172.178205$

Sum of electronic and thermal Free Energies=

$-1172.232290$

- Vertical excitation energies and oscillator strengths (gas phase)

$\begin{array}{llllll}\text { Excited State } & \text { 1: } & \text { Singlet-A } & 1.3369 \mathrm{eV} & 927.40 \mathrm{~nm} & \mathrm{f}=0.0023 \\ \text { Excited State } & \text { 2: } & \text { Singlet-A } & 1.4310 \mathrm{eV} & 866.40 \mathrm{~nm} & \mathrm{f}=0.0003 \\ \text { Excited State } & \text { 3: } & \text { Singlet-A } & 1.4875 \mathrm{eV} & 833.50 \mathrm{~nm} & \mathrm{f}=0.0000 \\ \text { Excited State } & \text { 4: } & \text { Singlet-A } & 1.7465 \mathrm{eV} & 709.90 \mathrm{~nm} & \mathrm{f}=0.0001 \\ \text { Excited State } & \text { 5: } & \text { Singlet-A } & 2.3496 \mathrm{eV} & 527.68 \mathrm{~nm} & \mathrm{f}=0.0000 \\ \text { Excited State } & \text { 6: } & \text { Singlet-A } & 2.3861 \mathrm{eV} & 519.61 \mathrm{~nm} & \mathrm{f}=0.0000 \\ \text { Excited State } & 7: & \text { Singlet-A } & 2.8025 \mathrm{eV} & 442.40 \mathrm{~nm} & \mathrm{f}=0.0004 \\ \text { Excited State } & \text { 8: } & \text { Singlet-A } & 2.8855 \mathrm{eV} & 429.68 \mathrm{~nm} & \mathrm{f}=0.0000 \\ \text { Excited State } & \text { 9: } & \text { Singlet-A } & 3.6386 \mathrm{eV} & 340.75 \mathrm{~nm} & \mathrm{f}=0.0039 \\ \text { Excited State } & 10: & \text { Singlet-A } & 3.7421 \mathrm{eV} & 331.32 \mathrm{~nm} & \mathrm{f}=0.0011 \\ \text { Excited State } & 11: & \text { Singlet-A } & 3.7802 \mathrm{eV} & 327.98 \mathrm{~nm} & \mathrm{f}=0.0000 \\ \text { Excited State } & 12: & \text { Singlet-A } & 3.9464 \mathrm{eV} & 314.17 \mathrm{~nm} & \mathrm{f}=0.7159 \\ \text { Excited State } & 13: & \text { Singlet-A } & 4.1348 \mathrm{eV} & 299.86 \mathrm{~nm} & \mathrm{f}=0.0017\end{array}$

- Vertical excitation energies and oscillator strengths (aqueous phase)

$\begin{array}{llllll}\text { Excited State } & \text { 1: } & \text { Singlet-A } & 3.0138 \mathrm{eV} & 411.40 \mathrm{~nm} & \mathrm{f}=0.0000 \\ \text { Excited State } & \text { 2: } & \text { Singlet-A } & 3.7196 \mathrm{eV} & 333.33 \mathrm{~nm} & \mathrm{f}=0.7416 \\ \text { Excited State } & \text { 3: } & \text { Singlet-A } & 3.8997 \mathrm{eV} & 317.93 \mathrm{~nm} & \mathrm{f}=0.0004 \\ \text { Excited State } & \text { 4: } & \text { Singlet-A } & 4.4090 \mathrm{eV} & 281.21 \mathrm{~nm} & \mathrm{f}=0.0000 \\ \text { Excited State } & \text { 5: } & \text { Singlet-A } & 4.8818 \mathrm{eV} & 253.97 \mathrm{~nm} & \mathrm{f}=0.0000 \\ \text { Excited State } & 6: & \text { Singlet-A } & 4.9029 \mathrm{eV} & 252.88 \mathrm{~nm} & \mathrm{f}=0.0001 \\ \text { Excited State } & 7: & \text { Singlet-A } & 5.1603 \mathrm{eV} & 240.26 \mathrm{~nm} & \mathrm{f}=0.0245 \\ \text { Excited State } & 8: & \text { Singlet-A } & 5.3390 \mathrm{eV} & 232.22 \mathrm{~nm} & \mathrm{f}=0.0109 \\ \text { Excited State } & 9: & \text { Singlet-A } & 5.3665 \mathrm{eV} & 231.03 \mathrm{~nm} & \mathrm{f}=0.0509 \\ \text { Excited State } & 10: & \text { Singlet-A } & 5.6546 \mathrm{eV} & 219.26 \mathrm{~nm} & \mathrm{f}=0.0002 \\ \text { Excited State } & 11: & \text { Singlet-A } & 5.7333 \mathrm{eV} & 216.25 \mathrm{~nm} & \mathrm{f}=0.0028 \\ \text { Excited State } & 12: & \text { Singlet-A } & 5.8850 \mathrm{eV} & 210.68 \mathrm{~nm} & \mathrm{f}=0.0003\end{array}$


Excited State 13: $\quad$ Singlet-A $\quad 5.9055 \mathrm{eV} \quad 209.95 \mathrm{~nm} \quad \mathrm{f}=0.0001$

\section{$\mathrm{Ca}^{2+}-p$-nitrophenol-NO${ }_{3}^{-}$}

- Cartesian coordinates:

\begin{tabular}{|c|c|c|c|}
\hline \multirow[t]{2}{*}{ Atomic number } & \multicolumn{3}{|c|}{ Coordinates (Angstroms) } \\
\hline & $\mathrm{X}$ & $\mathrm{Y}$ & $\mathrm{Z}$ \\
\hline 6 & -0.936756 & -0.204220 & -0.192534 \\
\hline 6 & 0.343070 & -0.608746 & -0.181026 \\
\hline 6 & 1.399217 & 0.366559 & -0.030328 \\
\hline 6 & 1.100552 & 1.774999 & 0.105789 \\
\hline 6 & -0.179084 & 2.178759 & 0.093842 \\
\hline 6 & -1.286498 & 1.218830 & -0.053837 \\
\hline 1 & -1.758981 & -0.899484 & -0.288306 \\
\hline 1 & 0.612394 & -1.649973 & -0.278321 \\
\hline 1 & 1.920608 & 2.469126 & 0.214701 \\
\hline 1 & -0.451983 & 3.220834 & 0.192815 \\
\hline 7 & 2.651348 & -0.033139 & -0.013927 \\
\hline 8 & 3.017847 & -1.305731 & -0.131475 \\
\hline 8 & 3.686775 & 0.790220 & 0.124031 \\
\hline 8 & -2.448853 & 1.607996 & -0.060190 \\
\hline 1 & -4.004350 & 0.770793 & -0.133375 \\
\hline 8 & -4.949257 & 0.481330 & -0.210102 \\
\hline 8 & -3.879152 & -1.379835 & 0.327279 \\
\hline 8 & -6.033392 & -1.382264 & 0.050945 \\
\hline 7 & -4.957734 & -0.867391 & 0.078969 \\
\hline 20 & 5.101613 & -0.822920 & 0.029092 \\
\hline
\end{tabular}

- Energies (a.u.):

Zero-point correction=

0.123682 (Hartree/Particle)

Thermal correction to Energy=

0.138201

Thermal correction to Enthalpy=

0.139145 
Thermal correction to Gibbs Free Energy=

Sum of electronic and zero-point Energies=

Sum of electronic and thermal Energies=

Sum of electronic and thermal Enthalpies=

Sum of electronic and thermal Free Energies=

$$
\begin{aligned}
& 0.077218 \\
& -1469.937738 \\
& -1469.923219 \\
& -1469.922275 \\
& -1469.984202
\end{aligned}
$$

- Vertical excitation energies and oscillator strengths (gas phase)

$\begin{array}{lcllll}\text { Excited State } & \text { 1: } & \text { Singlet-A } & 2.5984 \mathrm{eV} & 477.15 \mathrm{~nm} & \mathrm{f}=0.0000 \\ \text { Excited State } & \text { 2: } & \text { Singlet-A } & 2.7447 \mathrm{eV} & 451.73 \mathrm{~nm} & \mathrm{f}=0.0029 \\ \text { Excited State } & \text { 3: } & \text { Singlet-A } & 2.8877 \mathrm{eV} & 429.35 \mathrm{~nm} & \mathrm{f}=0.0005 \\ \text { Excited State } & \text { 4: } & \text { Singlet-A } & 3.0086 \mathrm{eV} & 412.09 \mathrm{~nm} & \mathrm{f}=0.0001 \\ \text { Excited State } & \text { 5: } & \text { Singlet-A } & 3.2918 \mathrm{eV} & 376.64 \mathrm{~nm} & \mathrm{f}=0.0000 \\ \text { Excited State } & 6: & \text { Singlet-A } & 3.5520 \mathrm{eV} & 349.05 \mathrm{~nm} & \mathrm{f}=0.0006 \\ \text { Excited State } & 7: & \text { Singlet-A } & 3.6345 \mathrm{eV} & 341.13 \mathrm{~nm} & \mathrm{f}=0.0085 \\ \text { Excited State } & 8: & \text { Singlet-A } & 3.6611 \mathrm{eV} & 338.65 \mathrm{~nm} & \mathrm{f}=0.0000 \\ \text { Excited State } & 9: & \text { Singlet-A } & 3.8701 \mathrm{eV} & 320.36 \mathrm{~nm} & \mathrm{f}=0.0002 \\ \text { Excited State } & 10: & \text { Singlet-A } & 3.8825 \mathrm{eV} & 319.34 \mathrm{~nm} & \mathrm{f}=0.7187 \\ \text { Excited State } & 11: & \text { Singlet-A } & 3.9018 \mathrm{eV} & 317.76 \mathrm{~nm} & \mathrm{f}=0.0000\end{array}$

- Vertical excitation energies and oscillator strengths (aqueous phase)

$\begin{array}{llllll}\text { Excited State } & \text { 1: } & \text { Singlet-A } & 3.2277 \mathrm{eV} & 384.13 \mathrm{~nm} & \mathrm{f}=0.0000 \\ \text { Excited State } & \text { 2: } & \text { Singlet-A } & 3.6526 \mathrm{eV} & 339.44 \mathrm{~nm} & \mathrm{f}=0.7688 \\ \text { Excited State } & \text { 3: } & \text { Singlet-A } & 3.9435 \mathrm{eV} & 314.40 \mathrm{~nm} & \mathrm{f}=0.0012 \\ \text { Excited State } & \text { 4: } & \text { Singlet-A } & 4.1377 \mathrm{eV} & 299.64 \mathrm{~nm} & \mathrm{f}=0.0009 \\ \text { Excited State } & \text { 5: } & \text { Singlet-A } & 4.1593 \mathrm{eV} & 298.09 \mathrm{~nm} & \mathrm{f}=0.0000 \\ \text { Excited State } & \text { 6: } & \text { Singlet-A } & 4.7613 \mathrm{eV} & 260.40 \mathrm{~nm} & \mathrm{f}=0.0007 \\ \text { Excited State } & 7: & \text { Singlet-A } & 4.7976 \mathrm{eV} & 258.43 \mathrm{~nm} & \mathrm{f}=0.0001 \\ \text { Excited State } & 8: & \text { Singlet-A } & 5.0450 \mathrm{eV} & 245.76 \mathrm{~nm} & \mathrm{f}=0.0001 \\ \text { Excited State } & \text { 9: } & \text { Singlet-A } & 5.1086 \mathrm{eV} & 242.70 \mathrm{~nm} & \mathrm{f}=0.0123 \\ \text { Excited State } & 10: & \text { Singlet-A } & 5.1236 \mathrm{eV} & 241.99 \mathrm{~nm} & \mathrm{f}=0.0003 \\ \text { Excited State } & 11: & \text { Singlet-A } & 5.2738 \mathrm{eV} & 235.10 \mathrm{~nm} & \mathrm{f}=0.0738\end{array}$

\section{$\underline{\mathrm{Ca}^{2+}-\text { p-nitrophenol-Cl- }}$}

- Cartesian coordinates:

\begin{tabular}{cccc}
\hline \multirow{2}{*}{ Atomic number } & \multicolumn{4}{c}{ Coordinates (Angstroms) } \\
\cline { 2 - 4 } & $\mathrm{X}$ & $\mathrm{Y}$ & $\mathrm{Z}$ \\
\hline 6 & 1.619160 & -0.403002 & -0.000010 \\
6 & 0.317739 & -0.729822 & -0.000010 \\
6 & -0.681158 & 0.315746 & -0.000004
\end{tabular}




$\begin{array}{rrrr}6 & -0.301060 & 1.711313 & -0.000001 \\ 6 & 1.000235 & 2.037518 & -0.000000 \\ 6 & 2.055182 & 1.006709 & -0.000003 \\ 1 & 2.394892 & -1.157312 & -0.000014 \\ 1 & -0.012973 & -1.757856 & -0.000015 \\ 1 & -1.080758 & 2.458473 & 0.000002 \\ 1 & 1.332516 & 3.067026 & 0.000003 \\ 7 & -1.954646 & -0.007007 & -0.000002 \\ 8 & -2.396412 & -1.262643 & -0.000005 \\ 8 & -2.942388 & 0.885803 & 0.000004 \\ 8 & 3.237211 & 1.320606 & 0.000002 \\ 1 & 4.596703 & -0.068730 & 0.000006 \\ 17 & 5.185960 & -1.225740 & 0.000006 \\ 20 & -4.447853 & -0.641794 & 0.000005\end{array}$

- Energies (a.u.):

Zero-point correction= 0.104812 (Hartree/Particle)

Thermal correction to Energy= 0.117021

Thermal correction to Enthalpy= 0.117966

Thermal correction to Gibbs Free Energy= 0.063183

Sum of electronic and zero-point Energies= $-1649.770919$

Sum of electronic and thermal Energies= $-1649.758710$

Sum of electronic and thermal Enthalpies= $-1649.757766$

Sum of electronic and thermal Free Energies= $-1649.812549$

- Vertical excitation energies and oscillator strengths (gas phase)

$\begin{array}{lcllll}\text { Excited State } & 1: & \text { Singlet-A } & 2.4441 \mathrm{eV} & 507.29 \mathrm{~nm} & \mathrm{f}=0.0000 \\ \text { Excited State } & \text { 2: } & \text { Singlet-A } & 2.7005 \mathrm{eV} & 459.11 \mathrm{~nm} & \mathrm{f}=0.0029 \\ \text { Excited State } & \text { 3: } & \text { Singlet-A } & 2.9643 \mathrm{eV} & 418.25 \mathrm{~nm} & \mathrm{f}=0.0000 \\ \text { Excited State } & \text { 4: } & \text { Singlet-A } & 2.9701 \mathrm{eV} & 417.44 \mathrm{~nm} & \mathrm{f}=0.0008 \\ \text { Excited State } & \text { 5: } & \text { Singlet-A } & 3.0390 \mathrm{eV} & 407.98 \mathrm{~nm} & \mathrm{f}=0.0000 \\ \text { Excited State } & \text { 6: } & \text { Singlet-A } & 3.0499 \mathrm{eV} & 406.52 \mathrm{~nm} & \mathrm{f}=0.0000 \\ \text { Excited State } & 7: & \text { Singlet-A } & 3.2416 \mathrm{eV} & 382.48 \mathrm{~nm} & \mathrm{f}=0.0000 \\ \text { Excited State } & 8: & \text { Singlet-A } & 3.6909 \mathrm{eV} & 335.92 \mathrm{~nm} & \mathrm{f}=0.0049 \\ \text { Excited State } & 9: & \text { Singlet-A } & 3.8576 \mathrm{eV} & 321.40 \mathrm{~nm} & \mathrm{f}=0.0000 \\ \text { Excited State } & 10: & \text { Singlet-A } & 3.9060 \mathrm{eV} & 317.42 \mathrm{~nm} & \mathrm{f}=0.7165 \\ & & & 26 & & \end{array}$


Excited State 11: $\quad$ Singlet-A $\quad 4.1677 \mathrm{eV} \quad 297.49 \mathrm{~nm} \quad \mathrm{f}=0.0043$

- Vertical excitation energies and oscillator strengths (aqueous phase)

$\begin{array}{llllll}\text { Excited State } & \text { 1: } & \text { Singlet-A } & 3.1262 \mathrm{eV} & 396.60 \mathrm{~nm} & \mathrm{f}=0.0000 \\ \text { Excited State } & \text { 2: } & \text { Singlet-A } & 3.6607 \mathrm{eV} & 338.69 \mathrm{~nm} & \mathrm{f}=0.7643 \\ \text { Excited State } & \text { 3: } & \text { Singlet-A } & 3.9824 \mathrm{eV} & 311.33 \mathrm{~nm} & \mathrm{f}=0.0009 \\ \text { Excited State } & \text { 4: } & \text { Singlet-A } & 4.1797 \mathrm{eV} & 296.64 \mathrm{~nm} & \mathrm{f}=0.0000 \\ \text { Excited State } & 5: & \text { Singlet-A } & 5.0290 \mathrm{eV} & 246.54 \mathrm{~nm} & \mathrm{f}=0.0000 \\ \text { Excited State } & 6: & \text { Singlet-A } & 5.0597 \mathrm{eV} & 245.04 \mathrm{~nm} & \mathrm{f}=0.0003 \\ \text { Excited State } & 7: & \text { Singlet-A } & 5.1077 \mathrm{eV} & 242.74 \mathrm{~nm} & \mathrm{f}=0.0069 \\ \text { Excited State } & 8: & \text { Singlet-A } & 5.1370 \mathrm{eV} & 241.35 \mathrm{~nm} & \mathrm{f}=0.0001 \\ \text { Excited State } & 9: & \text { Singlet-A } & 5.2545 \mathrm{eV} & 235.96 \mathrm{~nm} & \mathrm{f}=0.0774 \\ \text { Excited State } & 10: & \text { Singlet-A } & 5.6084 \mathrm{eV} & 221.07 \mathrm{~nm} & \mathrm{f}=0.0106 \\ \text { Excited State } & 11: & \text { Singlet-A } & 5.6487 \mathrm{eV} & 219.49 \mathrm{~nm} & \mathrm{f}=0.0002\end{array}$

$\underline{\mathrm{Na}}{ }^{+}-p$-nitrophenol-NO$\underline{3}^{-}$

- Cartesian coordinates:

\begin{tabular}{crrc}
\hline \multirow{2}{*}{ Atomic number } & \multicolumn{3}{c}{ Coordinates (Angstroms) } \\
\cline { 2 - 4 } & $\mathrm{X}$ & $\mathrm{Y}$ & $\mathrm{Z}$ \\
\hline 6 & -0.583012 & -0.250212 & -0.189536 \\
6 & 0.700019 & -0.685217 & -0.188529 \\
6 & 1.771671 & 0.240011 & -0.021549 \\
6 & 1.495625 & 1.627896 & 0.150714 \\
6 & 0.214117 & 2.066194 & 0.151611 \\
6 & -0.902624 & 1.155551 & -0.023377 \\
1 & -1.408345 & -0.940213 & -0.289626 \\
1 & 0.936179 & -1.732357 & -0.307948 \\
1 & 2.324690 & 2.308309 & 0.278227 \\
1 & -0.024966 & 3.113400 & 0.279880 \\
7 & 3.056466 & -0.201577 & -0.021101 \\
8 & 3.329807 & -1.441722 & -0.174002 \\
8 & 4.035388 & 0.608266 & 0.133174 \\
8 & -2.075888 & 1.587625 & -0.029576
\end{tabular}




$\begin{array}{rrrr}1 & -3.358680 & 0.785472 & -0.262505 \\ 8 & -4.295210 & 0.438518 & -0.522089 \\ 8 & -3.575150 & -1.206021 & 0.758428 \\ 8 & -5.570697 & -1.259241 & -0.094438 \\ 7 & -4.492075 & -0.756295 & 0.091878 \\ 11 & 5.510873 & -1.052204 & -0.003768\end{array}$

- Energies (a.u.):

Zero-point correction=

Thermal correction to Energy=

0.123277 (Hartree/Particle)

Thermal correction to Enthalpy=

0.137586

Thermal correction to Gibbs Free Energy=

0.138530

Sum of electronic and zero-point Energies=

0.077909

Sum of electronic and thermal Energies=

$-954.867309$

Sum of electronic and thermal Enthalpies=

$-954.853000$

$-954.852055$

Sum of electronic and thermal Free Energies=

$-954.912677$

- Vertical excitation energies and oscillator strengths (gas phase)

$\begin{array}{lcllll}\text { Excited State } & 1: & \text { Singlet-A } & 3.1962 \mathrm{eV} & 387.91 \mathrm{~nm} & \mathrm{f}=0.0000 \\ \text { Excited State } & \text { 2: } & \text { Singlet-A } & 3.2162 \mathrm{eV} & 385.50 \mathrm{~nm} & \mathrm{f}=0.0032 \\ \text { Excited State } & 3: & \text { Singlet-A } & 3.5966 \mathrm{eV} & 344.73 \mathrm{~nm} & \mathrm{f}=0.6479 \\ \text { Excited State } & \text { 4: } & \text { Singlet-A } & 3.8874 \mathrm{eV} & 318.94 \mathrm{~nm} & \mathrm{f}=0.0029 \\ \text { Excited State } & 5: & \text { Singlet-A } & 4.0775 \mathrm{eV} & 304.07 \mathrm{~nm} & \mathrm{f}=0.0000 \\ \text { Excited State } & \text { 6: } & \text { Singlet-A } & 4.1337 \mathrm{eV} & 299.94 \mathrm{~nm} & \mathrm{f}=0.0001 \\ \text { Excited State } & 7: & \text { Singlet-A } & 4.3653 \mathrm{eV} & 284.02 \mathrm{~nm} & \mathrm{f}=0.0000 \\ \text { Excited State } & 8: & \text { Singlet-A } & 4.6503 \mathrm{eV} & 266.61 \mathrm{~nm} & \mathrm{f}=0.0001 \\ \text { Excited State } & \text { 9: } & \text { Singlet-A } & 4.7286 \mathrm{eV} & 262.20 \mathrm{~nm} & \mathrm{f}=0.0000 \\ \text { Excited State } & 10: & \text { Singlet-A } & 4.7378 \mathrm{eV} & 261.69 \mathrm{~nm} & \mathrm{f}=0.0004\end{array}$

- Vertical excitation energies and oscillator strengths (aqueous phase)

$\begin{array}{llllll}\text { Excited State } & 1: & \text { Singlet-A } & 3.5067 \mathrm{eV} & 353.56 \mathrm{~nm} & \mathrm{f}=0.6602 \\ \text { Excited State } & 2: & \text { Singlet-A } & 3.6043 \mathrm{eV} & 343.99 \mathrm{~nm} & \mathrm{f}=0.0004 \\ \text { Excited State } & \text { 3: } & \text { Singlet-A } & 3.7013 \mathrm{eV} & 334.98 \mathrm{~nm} & \mathrm{f}=0.0141 \\ \text { Excited State } & \text { 4: } & \text { Singlet-A } & 3.8649 \mathrm{eV} & 320.79 \mathrm{~nm} & \mathrm{f}=0.0000 \\ \text { Excited State } & 5: & \text { Singlet-A } & 4.0872 \mathrm{eV} & 303.35 \mathrm{~nm} & \mathrm{f}=0.0033 \\ \text { Excited State } & 6: & \text { Singlet-A } & 4.5913 \mathrm{eV} & 270.04 \mathrm{~nm} & \mathrm{f}=0.0002 \\ \text { Excited State } & 7: & \text { Singlet-A } & 4.7780 \mathrm{eV} & 259.49 \mathrm{~nm} & \mathrm{f}=0.0012 \\ \text { Excited State } & 8: & \text { Singlet-A } & 4.8385 \mathrm{eV} & 256.24 \mathrm{~nm} & \mathrm{f}=0.0006 \\ \text { Excited State } & 9: & \text { Singlet-A } & 4.9061 \mathrm{eV} & 252.71 \mathrm{~nm} & \mathrm{f}=0.0371\end{array}$


Excited State $\quad 10: \quad$ Singlet-A $\quad 5.1726 \mathrm{eV} \quad 239.70 \mathrm{~nm} \quad \mathrm{f}=0.0001$

\section{$\underline{\mathrm{Na}^{+}-p \text {-nitrophenol-Cl}}$}

- Cartesian coordinates:

\begin{tabular}{|c|c|c|c|}
\hline \multirow[t]{2}{*}{ Atomic number } & \multicolumn{3}{|c|}{ Coordinates (Angstroms) } \\
\hline & $\mathrm{X}$ & Y & Z \\
\hline 6 & 1.215745 & -0.521550 & -0.000003 \\
\hline 6 & -0.101524 & -0.835789 & -0.000003 \\
\hline 6 & -1.087226 & 0.195242 & -0.000001 \\
\hline 6 & -0.687206 & 1.564355 & -0.000001 \\
\hline 6 & 0.628363 & 1.883728 & -0.000001 \\
\hline 6 & 1.661887 & 0.862355 & -0.000000 \\
\hline 1 & 1.974723 & -1.291862 & -0.000004 \\
\hline 1 & -0.432018 & -1.864107 & -0.000004 \\
\hline 1 & -1.452894 & 2.326172 & -0.000000 \\
\hline 1 & 0.960304 & 2.913350 & -0.000001 \\
\hline 7 & -2.405733 & -0.126064 & -0.000001 \\
\hline 8 & -2.790317 & -1.347278 & -0.000001 \\
\hline 8 & -3.309865 & 0.781342 & 0.000001 \\
\hline 8 & 2.869327 & 1.181596 & 0.000002 \\
\hline 1 & 3.942744 & 0.037918 & 0.000002 \\
\hline 17 & 4.829650 & -0.992220 & 0.000001 \\
\hline 11 & -4.926378 & -0.744236 & 0.000002 \\
\hline
\end{tabular}

- Energies (a.u.):

Zero-point correction=

Thermal correction to Energy=

0.104893 (Hartree/Particle)

0.116688

Thermal correction to Enthalpy=

0.117632

Thermal correction to Gibbs Free Energy=

0.064257

Sum of electronic and zero-point Energies=

$-1134.696788$

Sum of electronic and thermal Energies=

$-1134.684993$

Sum of electronic and thermal Enthalpies=

$-1134.684049$ 
- Vertical excitation energies and oscillator strengths (gas phase)

$\begin{array}{llllll}\text { Excited State } & \text { 1: } & \text { Singlet-A } & 3.0186 \mathrm{eV} & 410.74 \mathrm{~nm} & \mathrm{f}=0.0000 \\ \text { Excited State } & \text { 2: } & \text { Singlet-A } & 3.1678 \mathrm{eV} & 391.39 \mathrm{~nm} & \mathrm{f}=0.0033 \\ \text { Excited State } & \text { 3: } & \text { Singlet-A } & 3.5862 \mathrm{eV} & 345.73 \mathrm{~nm} & \mathrm{f}=0.6244 \\ \text { Excited State } & \text { 4: } & \text { Singlet-A } & 3.7791 \mathrm{eV} & 328.08 \mathrm{~nm} & \mathrm{f}=0.0296 \\ \text { Excited State } & \text { 5: } & \text { Singlet-A } & 3.7999 \mathrm{eV} & 326.29 \mathrm{~nm} & \mathrm{f}=0.0000 \\ \text { Excited State } & \text { 6: } & \text { Singlet-A } & 3.9324 \mathrm{eV} & 315.29 \mathrm{~nm} & \mathrm{f}=0.0033 \\ \text { Excited State } & \text { 7: } & \text { Singlet-A } & 4.0849 \mathrm{eV} & 303.52 \mathrm{~nm} & \mathrm{f}=0.0000 \\ \text { Excited State } & \text { 8: } & \text { Singlet-A } & 4.1234 \mathrm{eV} & 300.68 \mathrm{~nm} & \mathrm{f}=0.0000 \\ \text { Excited State } & \text { 9: } & \text { Singlet-A } & 4.4892 \mathrm{eV} & 276.18 \mathrm{~nm} & \mathrm{f}=0.0000 \\ \text { Excited State } & 10: & \text { Singlet-A } & 4.5393 \mathrm{eV} & 273.13 \mathrm{~nm} & \mathrm{f}=0.0002\end{array}$

- Vertical excitation energies and oscillator strengths (aqueous phase)

$\begin{array}{llllll}\text { Excited State } & \text { 1: } & \text { Singlet-A } & 3.5098 \mathrm{eV} & 353.25 \mathrm{~nm} & \mathrm{f}=0.6846 \\ \text { Excited State } & \text { 2: } & \text { Singlet-A } & 3.5424 \mathrm{eV} & 350.00 \mathrm{~nm} & \mathrm{f}=0.0000 \\ \text { Excited State } & \text { 3: } & \text { Singlet-A } & 3.8312 \mathrm{eV} & 323.62 \mathrm{~nm} & \mathrm{f}=0.0000 \\ \text { Excited State } & \text { 4: } & \text { Singlet-A } & 4.1075 \mathrm{eV} & 301.85 \mathrm{~nm} & \mathrm{f}=0.0032 \\ \text { Excited State } & \text { 5: } & \text { Singlet-A } & 4.8324 \mathrm{eV} & 256.57 \mathrm{~nm} & \mathrm{f}=0.0005 \\ \text { Excited State } & 6: & \text { Singlet-A } & 4.8916 \mathrm{eV} & 253.47 \mathrm{~nm} & \mathrm{f}=0.0352 \\ \text { Excited State } & 7: & \text { Singlet-A } & 5.1107 \mathrm{eV} & 242.60 \mathrm{~nm} & \mathrm{f}=0.0000 \\ \text { Excited State } & 8: & \text { Singlet-A } & 5.1154 \mathrm{eV} & 242.38 \mathrm{~nm} & \mathrm{f}=0.0005 \\ \text { Excited State } & \text { 9: } & \text { Singlet-A } & 5.4136 \mathrm{eV} & 229.02 \mathrm{~nm} & \mathrm{f}=0.0053 \\ \text { Excited State } & 10: & \text { Singlet-A } & 5.4877 \mathrm{eV} & 225.93 \mathrm{~nm} & \mathrm{f}=0.1052\end{array}$

\section{$\underline{\mathrm{K}}^{+}-$p-nitrophenol-NO${ }_{3}^{-}$}

- Cartesian coordinates:

\begin{tabular}{cccc}
\hline \multirow{2}{*}{ Atomic number } & \multicolumn{3}{c}{ Coordinates (Angstroms) } \\
\cline { 2 - 4 } & $\mathrm{X}$ & $\mathrm{Y}$ & $\mathrm{Z}$ \\
\hline 6 & -1.008215 & -0.196002 & -0.202935 \\
6 & 0.294872 & -0.574552 & -0.202431 \\
6 & 1.323222 & 0.392269 & -0.026272 \\
6 & 0.985806 & 1.761932 & 0.155838 \\
6 & -0.315737 & 2.144608 & 0.157372 \\
6 & -1.389622 & 1.190015 & -0.026696 \\
1 & -1.800323 & -0.923034 & -0.308935 \\
& & &
\end{tabular}




$\begin{array}{lrrr}1 & 0.575300 & -1.609850 & -0.329403 \\ 1 & 1.782944 & 2.478223 & 0.290689 \\ 1 & -0.599173 & 3.179768 & 0.293541 \\ 7 & 2.634488 & 0.004621 & -0.026162 \\ 8 & 2.951707 & -1.216594 & -0.188712 \\ 8 & 3.565908 & 0.856487 & 0.137507 \\ 8 & -2.584021 & 1.572428 & -0.031577 \\ 1 & -3.784878 & 0.710518 & -0.277140 \\ 8 & -4.697891 & 0.293901 & -0.555447 \\ 8 & -3.941723 & -1.256793 & 0.815875 \\ 8 & -5.899050 & -1.450265 & -0.102359 \\ 7 & -4.854852 & -0.881095 & 0.099161 \\ 19 & 5.519331 & -0.863329 & 0.004668\end{array}$

- Energies (a.u.):

Zero-point correction=

Thermal correction to Energy=

0.122607 (Hartree/Particle)
0.137188

Thermal correction to Enthalpy=

0.138133

Thermal correction to Gibbs Free Energy=

0.076006

Sum of electronic and zero-point Energies=

$-1392.510154$

Sum of electronic and thermal Energies=

$-1392.495573$

Sum of electronic and thermal Enthalpies=

$-1392.494629$

Sum of electronic and thermal Free Energies=

$-1392.556755$

- Vertical excitation energies and oscillator strengths (gas phase)

$\begin{array}{llllll}\text { Excited State } & \text { 1: } & \text { Singlet-A } & 3.2165 \mathrm{eV} & 385.47 \mathrm{~nm} & \mathrm{f}=0.0019 \\ \text { Excited State } & \text { 2: } & \text { Singlet-A } & 3.2726 \mathrm{eV} & 378.85 \mathrm{~nm} & \mathrm{f}=0.0000 \\ \text { Excited State } & \text { 3: } & \text { Singlet-A } & 3.5663 \mathrm{eV} & 347.65 \mathrm{~nm} & \mathrm{f}=0.6354 \\ \text { Excited State } & \text { 4: } & \text { Singlet-A } & 3.9228 \mathrm{eV} & 316.06 \mathrm{~nm} & \mathrm{f}=0.0026 \\ \text { Excited State } & 5: & \text { Singlet-A } & 4.0046 \mathrm{eV} & 309.60 \mathrm{~nm} & \mathrm{f}=0.0000 \\ \text { Excited State } & \text { 6: } & \text { Singlet-A } & 4.2115 \mathrm{eV} & 294.40 \mathrm{~nm} & \mathrm{f}=0.0001 \\ \text { Excited State } & 7: & \text { Singlet-A } & 4.3696 \mathrm{eV} & 283.74 \mathrm{~nm} & \mathrm{f}=0.0000 \\ \text { Excited State } & 8: & \text { Singlet-A } & 4.6236 \mathrm{eV} & 268.16 \mathrm{~nm} & \mathrm{f}=0.0001 \\ \text { Excited State } & 9: & \text { Singlet-A } & 4.6333 \mathrm{eV} & 267.59 \mathrm{~nm} & \mathrm{f}=0.0006 \\ \text { Excited State } & 10: & \text { Singlet-A } & 4.7298 \mathrm{eV} & 262.13 \mathrm{~nm} & \mathrm{f}=0.0000\end{array}$


- Vertical excitation energies and oscillator strengths (aqueous phase)

$\begin{array}{llllll}\text { Excited State } & \text { 1: } & \text { Singlet-A } & 3.4920 \mathrm{eV} & 355.05 \mathrm{~nm} & \mathrm{f}=0.6405 \\ \text { Excited State } & \text { 2: } & \text { Singlet-A } & 3.6353 \mathrm{eV} & 341.05 \mathrm{~nm} & \mathrm{f}=0.0002 \\ \text { Excited State } & \text { 3: } & \text { Singlet-A } & 3.6680 \mathrm{eV} & 338.02 \mathrm{~nm} & \mathrm{f}=0.0177 \\ \text { Excited State } & \text { 4: } & \text { Singlet-A } & 3.8821 \mathrm{eV} & 319.37 \mathrm{~nm} & \mathrm{f}=0.0000 \\ \text { Excited State } & \text { 5: } & \text { Singlet-A } & 4.1152 \mathrm{eV} & 301.28 \mathrm{~nm} & \mathrm{f}=0.0033 \\ \text { Excited State } & \text { 6: } & \text { Singlet-A } & 4.5660 \mathrm{eV} & 271.54 \mathrm{~nm} & \mathrm{f}=0.0002 \\ \text { Excited State } & 7: & \text { Singlet-A } & 4.6532 \mathrm{eV} & 266.45 \mathrm{~nm} & \mathrm{f}=0.0004 \\ \text { Excited State } & \text { 8: } & \text { Singlet-A } & 4.7924 \mathrm{eV} & 258.71 \mathrm{~nm} & \mathrm{f}=0.0015 \\ \text { Excited State } & 9: & \text { Singlet-A } & 4.8821 \mathrm{eV} & 253.96 \mathrm{~nm} & \mathrm{f}=0.0417 \\ \text { Excited State } & 10: & \text { Singlet-A } & 5.1511 \mathrm{eV} & 240.70 \mathrm{~nm} & \mathrm{f}=0.0001 \\ \text { Excited State } & 11: & \text { Singlet-A } & 5.3327 \mathrm{eV} & 232.50 \mathrm{~nm} & \mathrm{f}=0.0032\end{array}$

\section{$\underline{\mathrm{K}^{+}-p \text {-nitrophenol-Cl- }}$}

- Cartesian coordinates:

\begin{tabular}{|c|c|c|c|}
\hline \multirow[t]{2}{*}{ Atomic number } & \multicolumn{3}{|c|}{ Coordinates (Angstroms) } \\
\hline & $\mathrm{X}$ & $\mathrm{Y}$ & $\mathrm{Z}$ \\
\hline 6 & 1.638122 & -0.520470 & 0.000002 \\
\hline 6 & 0.306263 & -0.778727 & 0.000001 \\
\hline 6 & -0.633992 & 0.289298 & 0.000000 \\
\hline 6 & -0.177042 & 1.636767 & -0.000000 \\
\hline 6 & 1.153117 & 1.901292 & -0.000000 \\
\hline 6 & 2.140159 & 0.840165 & 0.000001 \\
\hline 1 & 2.362900 & -1.322933 & 0.000002 \\
\hline 1 & -0.065328 & -1.792954 & 0.000002 \\
\hline 1 & -0.908723 & 2.431355 & -0.000001 \\
\hline 1 & 1.526652 & 2.916620 & -0.000001 \\
\hline 7 & -1.973862 & 0.021620 & 0.000000 \\
\hline 8 & -2.398339 & -1.178470 & 0.000001 \\
\hline 8 & -2.827880 & 0.966380 & -0.000001 \\
\hline 8 & 3.364294 & 1.112733 & 0.000001 \\
\hline 1 & 4.338594 & -0.030297 & 0.000000 \\
\hline 17 & 5.203691 & -1.107285 & -0.000001 \\
\hline
\end{tabular}


- Energies (a.u.):

Zero-point correction=

Thermal correction to Energy=

0.104208 (Hartree/Particle)

Thermal correction to Enthalpy=

0.116255

Thermal correction to Gibbs Free Energy=

0.117199

Sum of electronic and zero-point Energies=

0.062376

Sum of electronic and thermal Energies=

$-1572.339421$

Sum of electronic and thermal Enthalpies=

$-1572.327374$

$-1572.326430$

Sum of electronic and thermal Free Energies=

$-1572.381252$

- Vertical excitation energies and oscillator strengths (gas phase)

$\begin{array}{llllll}\text { Excited State } & \text { 1: } & \text { Singlet-A } & 3.0732 \mathrm{eV} & 403.43 \mathrm{~nm} & \mathrm{f}=0.0000 \\ \text { Excited State } & \text { 2: } & \text { Singlet-A } & 3.1801 \mathrm{eV} & 389.87 \mathrm{~nm} & \mathrm{f}=0.0018 \\ \text { Excited State } & \text { 3: } & \text { Singlet-A } & 3.5460 \mathrm{eV} & 349.65 \mathrm{~nm} & \mathrm{f}=0.5812 \\ \text { Excited State } & \text { 4: } & \text { Singlet-A } & 3.7122 \mathrm{eV} & 333.99 \mathrm{~nm} & \mathrm{f}=0.0618 \\ \text { Excited State } & \text { 5: } & \text { Singlet-A } & 3.7238 \mathrm{eV} & 332.95 \mathrm{~nm} & \mathrm{f}=0.0000 \\ \text { Excited State } & \text { 6: } & \text { Singlet-A } & 3.9513 \mathrm{eV} & 313.78 \mathrm{~nm} & \mathrm{f}=0.0031 \\ \text { Excited State } & 7: & \text { Singlet-A } & 4.0049 \mathrm{eV} & 309.58 \mathrm{~nm} & \mathrm{f}=0.0000 \\ \text { Excited State } & \text { 8: } & \text { Singlet-A } & 4.1026 \mathrm{eV} & 302.21 \mathrm{~nm} & \mathrm{f}=0.0000 \\ \text { Excited State } & \text { 9: } & \text { Singlet-A } & 4.3706 \mathrm{eV} & 283.68 \mathrm{~nm} & \mathrm{f}=0.0000 \\ \text { Excited State } & 10: & \text { Singlet-A } & 4.4261 \mathrm{eV} & 280.12 \mathrm{~nm} & \mathrm{f}=0.0001\end{array}$

- Vertical excitation energies and oscillator strengths (aqueous phase)

$\begin{array}{lrllll}\text { Excited State } & \text { 1: } & \text { Singlet-A } & 3.4966 \mathrm{eV} & 354.59 \mathrm{~nm} & \mathrm{f}=0.6670 \\ \text { Excited State } & \text { 2: } & \text { Singlet-A } & 3.5994 \mathrm{eV} & 344.46 \mathrm{~nm} & \mathrm{f}=0.0000 \\ \text { Excited State } & \text { 3: } & \text { Singlet-A } & 3.8295 \mathrm{eV} & 323.76 \mathrm{~nm} & \mathrm{f}=0.0000 \\ \text { Excited State } & \text { 4: } & \text { Singlet-A } & 4.1248 \mathrm{eV} & 300.58 \mathrm{~nm} & \mathrm{f}=0.0034 \\ \text { Excited State } & \text { 5: } & \text { Singlet-A } & 4.6432 \mathrm{eV} & 267.02 \mathrm{~nm} & \mathrm{f}=0.0004 \\ \text { Excited State } & \text { 6: } & \text { Singlet-A } & 4.8802 \mathrm{eV} & 254.05 \mathrm{~nm} & \mathrm{f}=0.0397 \\ \text { Excited State } & \text { 7: } & \text { Singlet-A } & 5.0514 \mathrm{eV} & 245.45 \mathrm{~nm} & \mathrm{f}=0.0000 \\ \text { Excited State } & \text { 8: } & \text { Singlet-A } & 5.0573 \mathrm{eV} & 245.16 \mathrm{~nm} & \mathrm{f}=0.0005 \\ \text { Excited State } & \text { 9: } & \text { Singlet-A } & 5.3624 \mathrm{eV} & 231.21 \mathrm{~nm} & \mathrm{f}=0.0033 \\ \text { Excited State } & 10: & \text { Singlet-A } & 5.5516 \mathrm{eV} & 223.33 \mathrm{~nm} & \mathrm{f}=0.1084\end{array}$

\section{p-nitrophenolate}

- Cartesian coordinates:

\begin{tabular}{ll}
\hline Atomic number & Coordinates (Angstroms) \\
\hline
\end{tabular}




\begin{tabular}{cccc}
\hline & $\mathrm{X}$ & $\mathrm{Y}$ & $\mathrm{Z}$ \\
\hline 6 & 1.418899 & 1.222116 & -0.000000 \\
6 & 0.052342 & 1.217232 & -0.000000 \\
6 & -0.662631 & 0.000000 & -0.000000 \\
6 & 0.052341 & -1.217232 & 0.000001 \\
6 & 1.418898 & -1.222116 & -0.000000 \\
6 & 2.205690 & 0.000001 & -0.000000 \\
1 & 1.970143 & 2.155610 & -0.000000 \\
1 & -0.511040 & 2.140398 & -0.000000 \\
1 & -0.511042 & -2.140398 & 0.000001 \\
1 & 1.970144 & -2.155608 & 0.000001 \\
7 & -2.074032 & 0.000000 & -0.000000 \\
8 & -2.684814 & 1.087042 & -0.000000 \\
8 & -2.684812 & -1.087043 & -0.000000 \\
8 & 3.455474 & -0.000001 & -0.000000
\end{tabular}

- Energies (a.u.):

Zero-point correction=

0.094022 (Hartree/Particle)

Thermal correction to Energy=

0.101610

Thermal correction to Enthalpy=

0.102554

Thermal correction to Gibbs Free Energy=

0.061169

Sum of electronic and zero-point Energies=

$-511.571058$

Sum of electronic and thermal Energies $=$

$-511.563470$

$-511.562526$

Sum of electronic and thermal Enthalpies=

Sum of electronic and thermal Free Energies=

$-511.603911$

- Vertical excitation energies and oscillator strengths (gas phase)

$\begin{array}{llllll}\text { Excited State } & \text { 1: } & \text { Singlet-A } & 3.2720 \mathrm{eV} & 378.92 \mathrm{~nm} & \mathrm{f}=0.0000 \\ \text { Excited State } & \text { 2: } & \text { Singlet-A } & 3.6034 \mathrm{eV} & 344.07 \mathrm{~nm} & \mathrm{f}=0.4369 \\ \text { Excited State } & \text { 3: } & \text { Singlet-A } & 3.8992 \mathrm{eV} & 317.97 \mathrm{~nm} & \mathrm{f}=0.0000 \\ \text { Excited State } & \text { 4: } & \text { Singlet-A } & 4.4154 \mathrm{eV} & 280.80 \mathrm{~nm} & \mathrm{f}=0.0323 \\ \text { Excited State } & \text { 5: } & \text { Singlet-A } & 4.5697 \mathrm{eV} & 271.32 \mathrm{~nm} & \mathrm{f}=0.0134 \\ \text { Excited State } & 6: & \text { Singlet-A } & 4.6376 \mathrm{eV} & 267.34 \mathrm{~nm} & \mathrm{f}=0.0003 \\ \text { Excited State } & 7: & \text { Singlet-A } & 4.7852 \mathrm{eV} & 259.10 \mathrm{~nm} & \mathrm{f}=0.0001 \\ \text { Excited State } & 8: & \text { Singlet-A } & 5.7521 \mathrm{eV} & 215.54 \mathrm{~nm} & \mathrm{f}=0.0012\end{array}$




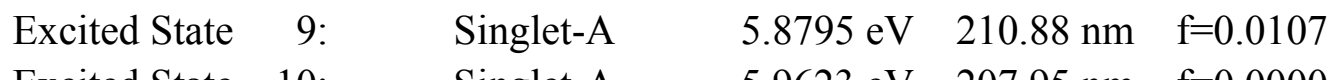

Excited State 10: $\quad$ Singlet-A $\quad 5.9623 \mathrm{eV} \quad 207.95 \mathrm{~nm} \quad \mathrm{f}=0.0000$

- Vertical excitation energies and oscillator strengths (aqueous phase)

$\begin{array}{llllll}\text { Excited State } & \text { 1: } & \text { Singlet-A } & 3.3935 \mathrm{eV} & 365.36 \mathrm{~nm} & \mathrm{f}=0.0000 \\ \text { Excited State } & \text { 2: } & \text { Singlet-A } & 3.4676 \mathrm{eV} & 357.55 \mathrm{~nm} & \mathrm{f}=0.5286 \\ \text { Excited State } & \text { 3: } & \text { Singlet-A } & 3.9432 \mathrm{eV} & 314.43 \mathrm{~nm} & \mathrm{f}=0.0000 \\ \text { Excited State } & \text { 4: } & \text { Singlet-A } & 4.3595 \mathrm{eV} & 284.40 \mathrm{~nm} & \mathrm{f}=0.0001 \\ \text { Excited State } & \text { 5: } & \text { Singlet-A } & 4.5733 \mathrm{eV} & 271.11 \mathrm{~nm} & \mathrm{f}=0.0600 \\ \text { Excited State } & 6: & \text { Singlet-A } & 4.6873 \mathrm{eV} & 264.51 \mathrm{~nm} & \mathrm{f}=0.0003 \\ \text { Excited State } & 7: & \text { Singlet-A } & 5.0662 \mathrm{eV} & 244.73 \mathrm{~nm} & \mathrm{f}=0.0002 \\ \text { Excited State } & 8: & \text { Singlet-A } & 5.9034 \mathrm{eV} & 210.02 \mathrm{~nm} & \mathrm{f}=0.0153 \\ \text { Excited State } & 9: & \text { Singlet-A } & 6.0466 \mathrm{eV} & 205.05 \mathrm{~nm} & \mathrm{f}=0.1104 \\ \text { Excited State } & 10: & \text { Singlet-A } & 6.1280 \mathrm{eV} & 202.32 \mathrm{~nm} & \mathrm{f}=0.0000\end{array}$

$\underline{M^{2+}-p-n i t r o p h e n o l a t e}$

$\mathrm{Mg}^{2+}-p$-nitrophenolate (a)

- Cartesian coordinates:

\begin{tabular}{lccc}
\hline \multirow{2}{*}{ Atomic number } & \multicolumn{3}{c}{ Coordinates (Angstroms) } \\
\cline { 2 - 4 } & $\mathrm{X}$ & $\mathrm{Y}$ & $\mathrm{Z}$ \\
\hline 6 & 0.693777 & 1.220913 & 0.145562 \\
6 & -0.682300 & 1.220431 & 0.060593 \\
6 & -1.353361 & -0.000000 & 0.017222 \\
6 & -0.682301 & -1.220431 & 0.060587 \\
6 & 0.693777 & -1.220914 & 0.145556 \\
6 & 1.392993 & -0.000001 & 0.213134 \\
1 & 1.247716 & 2.149381 & 0.183459 \\
1 & -1.247864 & 2.139889 & 0.024785 \\
1 & -1.247865 & -2.139889 & 0.024774 \\
1 & 1.247716 & -2.149382 & 0.183447 \\
7 & -2.831630 & 0.000000 & -0.091909 \\
8 & -3.383698 & 1.084655 & -0.133019 \\
8 & -3.383700 & -1.084654 & -0.133004
\end{tabular}


8

$0.000001-0.397632$
- Energies (a.u.):

Zero-point correction=

Thermal correction to Energy=

Thermal correction to Enthalpy=

Thermal correction to Gibbs Free Energy=

Sum of electronic and zero-point Energies=

Sum of electronic and thermal Energies=

Sum of electronic and thermal Enthalpies=

Sum of electronic and thermal Free Energies=

0.096074 (Hartree/Particle)
0.105521
0.106465
0.058722
-711.327665
-711.318218
-711.317274
-711.365017

- Vertical excitation energies and oscillator strengths (gas phase)

\begin{tabular}{|c|c|c|c|c|c|}
\hline Excited State & 1: & Singlet-A & $1.1204 \mathrm{eV}$ & $106.60 \mathrm{~nm}$ & $\mathrm{f}=0.0000$ \\
\hline Excited State & 2: & Singlet-A & $1.2096 \mathrm{eV}$ & $024.98 \mathrm{~nm}$ & $\mathrm{f}=0.0008$ \\
\hline Excited State & 3: & Singlet-A & $1.2276 \mathrm{eV}$ & $009.95 \mathrm{~nm}$ & $\mathrm{f}=0.1761$ \\
\hline Excited State & 4: & Singlet-A & $1.5082 \mathrm{eV}$ & $822.06 \mathrm{~nm}$ & $\mathrm{f}=0.0021$ \\
\hline Excited State & 5: & Singlet-A & $1.6801 \mathrm{eV}$ & $737.98 \mathrm{~nm}$ & $\mathrm{f}=0.0005$ \\
\hline Excited State & $6:$ & Singlet-A & $2.3929 \mathrm{eV}$ & $518.13 \mathrm{~nm}$ & $\mathrm{f}=0.0025$ \\
\hline Excited State & 7: & Singlet-A & $3.6608 \mathrm{eV}$ & $338.68 \mathrm{~nm}$ & $\mathrm{f}=0.0077$ \\
\hline Excited State & 8: & Singlet-A & $3.8873 \mathrm{eV}$ & $318.95 \mathrm{~nm}$ & $\mathrm{f}=0.0000$ \\
\hline Excited State & 9: & Singlet-A & $3.9625 \mathrm{eV}$ & $312.89 \mathrm{~nm}$ & $\mathrm{f}=0.0001$ \\
\hline Excited State & 10: & Singlet-A & $4.1891 \mathrm{eV}$ & $295.97 \mathrm{~nm}$ & $\mathrm{f}=0.0678$ \\
\hline Excited State & 11: & Singlet-A & $4.2513 \mathrm{eV}$ & $291.64 \mathrm{~nm}$ & $f=0.0746$ \\
\hline Excited State & 12: & Singlet-A & $4.3394 \mathrm{eV}$ & $285.72 \mathrm{~nm}$ & $\mathrm{f}=0.0002$ \\
\hline Excited State & 13: & Singlet-A & $4.7508 \mathrm{eV}$ & $260.98 \mathrm{~nm}$ & $\mathrm{f}=0.2482$ \\
\hline Excited State & 14: & Singlet-A & $4.8464 \mathrm{eV}$ & $255.83 \mathrm{~nm}$ & $\mathrm{f}=0.001$ \\
\hline
\end{tabular}

- Vertical excitation energies and oscillator strengths (aqueous phase)

$\begin{array}{llllll}\text { Excited State } & \text { 1: } & \text { Singlet-A } & 3.6941 \mathrm{eV} & 335.63 \mathrm{~nm} & \mathrm{f}=0.3727 \\ \text { Excited State } & \text { 2: } & \text { Singlet-A } & 4.2004 \mathrm{eV} & 295.17 \mathrm{~nm} & \mathrm{f}=0.0000 \\ \text { Excited State } & \text { 3: } & \text { Singlet-A } & 4.3173 \mathrm{eV} & 287.18 \mathrm{~nm} & \mathrm{f}=0.0042 \\ \text { Excited State } & \text { 4: } & \text { Singlet-A } & 4.4455 \mathrm{eV} & 278.90 \mathrm{~nm} & \mathrm{f}=0.0054 \\ \text { Excited State } & \text { 5: } & \text { Singlet-A } & 4.6024 \mathrm{eV} & 269.39 \mathrm{~nm} & \mathrm{f}=0.0002 \\ \text { Excited State } & \text { 6: } & \text { Singlet-A } & 4.6646 \mathrm{eV} & 265.80 \mathrm{~nm} & \mathrm{f}=0.0000 \\ \text { Excited State } & 7: & \text { Singlet-A } & 5.0592 \mathrm{eV} & 245.06 \mathrm{~nm} & \mathrm{f}=0.0613 \\ \text { Excited State } & 8: & \text { Singlet-A } & 5.5309 \mathrm{eV} & 224.17 \mathrm{~nm} & \mathrm{f}=0.0240 \\ \text { Excited State } & \text { 9: } & \text { Singlet-A } & 5.6974 \mathrm{eV} & 217.61 \mathrm{~nm} & \mathrm{f}=0.0013 \\ \text { Excited State } & 10: & \text { Singlet-A } & 6.0217 \mathrm{eV} & 205.90 \mathrm{~nm} & \mathrm{f}=0.0010\end{array}$


$\mathrm{Mg}^{2+}-p$-nitrophenolate (b)

- Cartesian coordinates:

\begin{tabular}{crrc}
\hline \multirow{2}{*}{ tomic number } & \multicolumn{3}{c}{ Coordinates (Angstroms) } \\
\cline { 2 - 4 } & $\mathrm{X}$ & $\mathrm{Y}$ & $\mathrm{Z}$ \\
\hline 6 & 2.017825 & 1.260822 & 0.000002 \\
6 & 0.677330 & 1.263271 & 0.000002 \\
6 & -0.032221 & -0.000000 & 0.000002 \\
6 & 0.677331 & -1.263271 & 0.000002 \\
6 & 2.017826 & -1.260822 & 0.000002 \\
6 & 2.793681 & 0.000000 & -0.000001 \\
1 & 2.591268 & 2.178350 & 0.000002 \\
1 & 0.101802 & 2.177065 & 0.000004 \\
1 & 0.101803 & -2.177065 & 0.000004 \\
1 & 2.591269 & -2.178350 & 0.000002 \\
7 & -1.341617 & -0.000001 & 0.000000 \\
8 & -2.093222 & 1.121536 & -0.000000 \\
8 & -2.093221 & -1.121536 & -0.000000 \\
8 & 4.008704 & 0.000000 & -0.000005 \\
12 & -3.623629 & 0.000000 & -0.000000
\end{tabular}

- Energies (a.u.):

Zero-point correction=

Thermal correction to Energy= 0.096272 (Hartree/Particle)

Thermal correction to Enthalpy=

0.105377

Thermal correction to Gibbs Free Energy=

0.106321

Sum of electronic and zero-point Energies= 0.061192

Sum of electronic and thermal Energies=

$-711.356270$

$-711.347165$

Sum of electronic and thermal Enthalpies=

$-711.346221$

Sum of electronic and thermal Free Energies=

$-711.391351$

- Vertical excitation energies and oscillator strengths (gas phase)

$\begin{array}{llllll}\text { Excited State } & 1: & \text { Singlet-A } & 1.2634 \mathrm{eV} & 981.35 \mathrm{~nm} & \mathrm{f}=0.0023 \\ \text { Excited State } & 2: & \text { Singlet-A } & 1.3823 \mathrm{eV} & 896.97 \mathrm{~nm} & \mathrm{f}=0.0000\end{array}$




$\begin{array}{lrllll}\text { Excited State } & \text { 3: } & \text { Singlet-A } & 2.2201 \mathrm{eV} & 558.47 \mathrm{~nm} & \mathrm{f}=0.0000 \\ \text { Excited State } & \text { 4: } & \text { Singlet-A } & 2.3560 \mathrm{eV} & 526.26 \mathrm{~nm} & \mathrm{f}=0.0000 \\ \text { Excited State } & \text { 5: } & \text { Singlet-A } & 3.7066 \mathrm{eV} & 334.50 \mathrm{~nm} & \mathrm{f}=0.0006 \\ \text { Excited State } & \text { 6: } & \text { Singlet-A } & 3.7284 \mathrm{eV} & 332.54 \mathrm{~nm} & \mathrm{f}=0.0029 \\ \text { Excited State } & \text { 7: } & \text { Singlet-A } & 3.7371 \mathrm{eV} & 331.76 \mathrm{~nm} & \mathrm{f}=0.0000 \\ \text { Excited State } & \text { 8: } & \text { Singlet-A } & 3.9814 \mathrm{eV} & 311.41 \mathrm{~nm} & \mathrm{f}=0.6783 \\ \text { Excited State } & \text { 9: } & \text { Singlet-A } & 4.0329 \mathrm{eV} & 307.43 \mathrm{~nm} & \mathrm{f}=0.0017 \\ \text { Excited State } & 10: & \text { Singlet-A } & 4.6388 \mathrm{eV} & 267.28 \mathrm{~nm} & \mathrm{f}=0.0003\end{array}$

- Vertical excitation energies and oscillator strengths (aqueous phase)

$\begin{array}{llllll}\text { Excited State } & \text { 1: } & \text { Singlet-A } & 2.8522 \mathrm{eV} & 434.69 \mathrm{~nm} & \mathrm{f}=0.0000 \\ \text { Excited State } & \text { 2: } & \text { Singlet-A } & 3.7521 \mathrm{eV} & 330.44 \mathrm{~nm} & \mathrm{f}=0.7068 \\ \text { Excited State } & \text { 3: } & \text { Singlet-A } & 3.9519 \mathrm{eV} & 313.74 \mathrm{~nm} & \mathrm{f}=0.0000 \\ \text { Excited State } & \text { 4: } & \text { Singlet-A } & 4.4694 \mathrm{eV} & 277.40 \mathrm{~nm} & \mathrm{f}=0.0000 \\ \text { Excited State } & \text { 5: } & \text { Singlet-A } & 5.1854 \mathrm{eV} & 239.10 \mathrm{~nm} & \mathrm{f}=0.0180 \\ \text { Excited State } & 6: & \text { Singlet-A } & 5.2510 \mathrm{eV} & 236.12 \mathrm{~nm} & \mathrm{f}=0.0112 \\ \text { Excited State } & 7: & \text { Singlet-A } & 5.3476 \mathrm{eV} & 231.85 \mathrm{~nm} & \mathrm{f}=0.0551 \\ \text { Excited State } & 8: & \text { Singlet-A } & 5.4644 \mathrm{eV} & 226.89 \mathrm{~nm} & \mathrm{f}=0.0002 \\ \text { Excited State } & 9: & \text { Singlet-A } & 5.7506 \mathrm{eV} & 215.60 \mathrm{~nm} & \mathrm{f}=0.0028 \\ \text { Excited State } & 10: & \text { Singlet-A } & 5.8769 \mathrm{eV} & 210.97 \mathrm{~nm} & \mathrm{f}=0.0000\end{array}$

\section{$\underline{C a^{2+}-p \text {-nitrophenolate }}$}

$\mathrm{Ca}^{2+}-p$-nitrophenolate (a)

- Cartesian coordinates:

\begin{tabular}{cccc}
\hline \multirow{2}{*}{ Atomic number } & \multicolumn{3}{c}{ Coordinates (Angstroms) } \\
\cline { 2 - 4 } & $\mathrm{X}$ & $\mathrm{Y}$ & $\mathrm{Z}$ \\
\hline 6 & 0.242804 & 1.210926 & -0.000003 \\
6 & -1.142209 & 1.212677 & -0.000003 \\
6 & -1.817416 & -0.000000 & -0.000000 \\
6 & -1.142209 & -1.212678 & 0.000001 \\
6 & 0.242804 & -1.210927 & 0.000001 \\
6 & 0.935150 & -0.000000 & -0.000001 \\
1 & 0.794177 & 2.141964 & -0.000005 \\
1 & -1.703415 & 2.135344 & -0.000004 \\
1 & -1.703416 & -2.135344 & 0.000003 \\
1 & 0.794176 & -2.141964 & 0.000003
\end{tabular}




$\begin{array}{rrrr}7 & -3.294909 & 0.000000 & 0.000001 \\ 8 & -3.855586 & 1.082982 & 0.000006 \\ 8 & -3.855586 & -1.082981 & -0.000004 \\ 8 & 2.294201 & -0.000001 & -0.000001 \\ 20 & 4.215253 & 0.000000 & 0.000001\end{array}$

- Energies (a.u.):

Zero-point correction=

Thermal correction to Energy=

0.096452 (Hartree/Particle)

Thermal correction to Enthalpy=

0.105864

Thermal correction to Gibbs Free Energy=

0.106808

Sum of electronic and zero-point Energies=

0.059390

$-1188.921915$

Sum of electronic and thermal Energies $=$

$-1188.912503$

Sum of electronic and thermal Enthalpies=

$-1188.911559$

Sum of electronic and thermal Free Energies=

$-1188.958977$

- Vertical excitation energies and oscillator strengths (gas phase)

$\begin{array}{llllll}\text { Excited State } & \text { 1: } & \text { Singlet-A } & 1.7148 \mathrm{eV} & 723.03 \mathrm{~nm} & \mathrm{f}=0.0002 \\ \text { Excited State } & \text { 2: } & \text { Singlet-A } & 2.3310 \mathrm{eV} & 531.90 \mathrm{~nm} & \mathrm{f}=0.0000 \\ \text { Excited State } & \text { 3: } & \text { Singlet-A } & 2.4289 \mathrm{eV} & 510.46 \mathrm{~nm} & \mathrm{f}=0.0000 \\ \text { Excited State } & \text { 4: } & \text { Singlet-A } & 2.8465 \mathrm{eV} & 435.57 \mathrm{~nm} & \mathrm{f}=0.0004 \\ \text { Excited State } & \text { 5: } & \text { Singlet-A } & 2.9579 \mathrm{eV} & 419.17 \mathrm{~nm} & \mathrm{f}=0.0000 \\ \text { Excited State } & 6: & \text { Singlet-A } & 3.2874 \mathrm{eV} & 377.15 \mathrm{~nm} & \mathrm{f}=0.0002 \\ \text { Excited State } & 7: & \text { Singlet-A } & 3.2877 \mathrm{eV} & 377.11 \mathrm{~nm} & \mathrm{f}=0.0001 \\ \text { Excited State } & 8: & \text { Singlet-A } & 3.6167 \mathrm{eV} & 342.81 \mathrm{~nm} & \mathrm{f}=0.0030 \\ \text { Excited State } & 9: & \text { Singlet-A } & 3.6579 \mathrm{eV} & 338.95 \mathrm{~nm} & \mathrm{f}=0.0000 \\ \text { Excited State } & 10: & \text { Singlet-A } & 3.7769 \mathrm{eV} & 328.27 \mathrm{~nm} & \mathrm{f}=0.0000 \\ \text { Excited State } & 11: & \text { Singlet-A } & 3.7844 \mathrm{eV} & 327.62 \mathrm{~nm} & \mathrm{f}=0.1017 \\ \text { Excited State } & 12: & \text { Singlet-A } & 3.8768 \mathrm{eV} & 319.81 \mathrm{~nm} & \mathrm{f}=0.0040\end{array}$

- Vertical excitation energies and oscillator strengths (aqueous phase)

$\begin{array}{lllrll}\text { Excited State } & \text { 1: } & \text { Singlet-A } & 3.6858 \mathrm{eV} & 336.38 \mathrm{~nm} & \mathrm{f}=0.3915 \\ \text { Excited State } & \text { 2: } & \text { Singlet-A } & 4.1388 \mathrm{eV} & 299.56 \mathrm{~nm} & \mathrm{f}=0.0000 \\ \text { Excited State } & \text { 3: } & \text { Singlet-A } & 4.2493 \mathrm{eV} & 291.77 \mathrm{~nm} & \mathrm{f}=0.0055 \\ \text { Excited State } & \text { 4: } & \text { Singlet-A } & 4.5954 \mathrm{eV} & 269.80 \mathrm{~nm} & \mathrm{f}=0.0002 \\ \text { Excited State } & \text { 5: } & \text { Singlet-A } & 4.6222 \mathrm{eV} & 268.23 \mathrm{~nm} & \mathrm{f}=0.0000 \\ \text { Excited State } & \text { 6: } & \text { Singlet-A } & 5.0577 \mathrm{eV} & 245.14 \mathrm{~nm} & \mathrm{f}=0.0596 \\ \text { Excited State } & \text { 7: } & \text { Singlet-A } & 5.2316 \mathrm{eV} & 236.99 \mathrm{~nm} & \mathrm{f}=0.0016 \\ \text { Excited State } & \text { 8: } & \text { Singlet-A } & 6.0124 \mathrm{eV} & 206.21 \mathrm{~nm} & \mathrm{f}=0.0005 \\ \text { Excited State } & \text { 9: } & \text { Singlet-A } & 6.0470 \mathrm{eV} & 205.03 \mathrm{~nm} & \mathrm{f}=0.0146 \\ & & & & & \end{array}$


Excited State 10: $\quad$ Singlet-A $\quad 6.2275 \mathrm{eV} \quad 199.09 \mathrm{~nm} \quad \mathrm{f}=0.0465$

$\mathrm{Ca}^{2+}-p$-nitrophenolate (b)

- Cartesian coordinates:

\begin{tabular}{cccc}
\hline \multirow{2}{*}{ Atomic number } & \multicolumn{3}{c}{ Coordinates (Angstroms) } \\
\cline { 2 - 4 } & $\mathrm{X}$ & $\mathrm{Y}$ & $\mathrm{Z}$ \\
\hline 6 & 2.400272 & 1.258698 & 0.000000 \\
6 & 1.058985 & 1.260257 & 0.000002 \\
6 & 0.347561 & 0.000000 & 0.000003 \\
6 & 1.058985 & -1.260256 & 0.000003 \\
6 & 2.400272 & -1.258698 & 0.000000 \\
6 & 3.176694 & -0.000000 & -0.000001 \\
1 & 2.972940 & 2.176644 & -0.000000 \\
1 & 0.484315 & 2.174587 & 0.000004 \\
1 & 0.484314 & -2.174587 & 0.000004 \\
1 & 2.972940 & -2.176645 & -0.000000 \\
7 & -0.964995 & 0.000000 & 0.000002 \\
8 & -1.705008 & 1.110137 & 0.000001 \\
8 & -1.705007 & -1.110137 & 0.000001 \\
8 & 4.393324 & -0.000000 & -0.000004 \\
\hline & -3.534131 & -0.000000 & -0.000002
\end{tabular}

- Energies (a.u.):

Zero-point correction $=$ 0.095806 (Hartree/Particle)

Thermal correction to Energy= 0.105127

Thermal correction to Enthalpy=

0.106071

Thermal correction to Gibbs Free Energy= 0.060036

Sum of electronic and zero-point Energies= $-1188.935238$

Sum of electronic and thermal Energies= $-1188.925918$

Sum of electronic and thermal Enthalpies= $-1188.924974$

Sum of electronic and thermal Free Energies $=$

$-1188.971008$

- Vertical excitation energies and oscillator strengths (gas phase) 


$\begin{array}{lcllll}\text { Excited State } & \text { 1: } & \text { Singlet-A } & 2.2908 \mathrm{eV} & 541.23 \mathrm{~nm} & \mathrm{f}=0.0000 \\ \text { Excited State } & \text { 2: } & \text { Singlet-A } & 2.6090 \mathrm{eV} & 475.21 \mathrm{~nm} & \mathrm{f}=0.0028 \\ \text { Excited State } & \text { 3: } & \text { Singlet-A } & 2.8197 \mathrm{eV} & 439.71 \mathrm{~nm} & \mathrm{f}=0.0000 \\ \text { Excited State } & \text { 4: } & \text { Singlet-A } & 3.7881 \mathrm{eV} & 327.30 \mathrm{~nm} & \mathrm{f}=0.0014 \\ \text { Excited State } & \text { 5: } & \text { Singlet-A } & 3.8135 \mathrm{eV} & 325.12 \mathrm{~nm} & \mathrm{f}=0.0000 \\ \text { Excited State } & 6: & \text { Singlet-A } & 3.9534 \mathrm{eV} & 313.62 \mathrm{~nm} & \mathrm{f}=0.6760 \\ \text { Excited State } & 7: & \text { Singlet-A } & 4.0918 \mathrm{eV} & 303.01 \mathrm{~nm} & \mathrm{f}=0.0047 \\ \text { Excited State } & 8: & \text { Singlet-A } & 4.1976 \mathrm{eV} & 295.37 \mathrm{~nm} & \mathrm{f}=0.0006 \\ \text { Excited State } & \text { 9: } & \text { Singlet-A } & 4.2967 \mathrm{eV} & 288.55 \mathrm{~nm} & \mathrm{f}=0.0057 \\ \text { Excited State } & 10: & \text { Singlet-A } & 4.2997 \mathrm{eV} & 288.36 \mathrm{~nm} & \mathrm{f}=0.0010\end{array}$

- Vertical excitation energies and oscillator strengths (aqueous phase)

$\begin{array}{llllll}\text { Excited State } & \text { 1: } & \text { Singlet-A } & 2.9410 \mathrm{eV} & 421.56 \mathrm{~nm} & \mathrm{f}=0.0000 \\ \text { Excited State } & \text { 2: } & \text { Singlet-A } & 3.6943 \mathrm{eV} & 335.61 \mathrm{~nm} & \mathrm{f}=0.7259 \\ \text { Excited State } & \text { 3: } & \text { Singlet-A } & 4.0419 \mathrm{eV} & 306.75 \mathrm{~nm} & \mathrm{f}=0.0003 \\ \text { Excited State } & \text { 4: } & \text { Singlet-A } & 4.2385 \mathrm{eV} & 292.52 \mathrm{~nm} & \mathrm{f}=0.0000 \\ \text { Excited State } & 5: & \text { Singlet-A } & 5.1119 \mathrm{eV} & 242.54 \mathrm{~nm} & \mathrm{f}=0.0007 \\ \text { Excited State } & 6: & \text { Singlet-A } & 5.1730 \mathrm{eV} & 239.68 \mathrm{~nm} & \mathrm{f}=0.0001 \\ \text { Excited State } & 7: & \text { Singlet-A } & 5.2428 \mathrm{eV} & 236.49 \mathrm{~nm} & \mathrm{f}=0.0812 \\ \text { Excited State } & 8: & \text { Singlet-A } & 5.4305 \mathrm{eV} & 228.31 \mathrm{~nm} & \mathrm{f}=0.0002 \\ \text { Excited State } & \text { 9: } & \text { Singlet-A } & 5.5118 \mathrm{eV} & 224.94 \mathrm{~nm} & \mathrm{f}=0.0112 \\ \text { Excited State } & 10: & \text { Singlet-A } & 5.7654 \mathrm{eV} & 215.05 \mathrm{~nm} & \mathrm{f}=0.0022\end{array}$

\section{$\underline{N a^{+}-p-n i t r o p h e n o l a t e}$}

$\mathrm{Na}^{+}-p$-nitrophenolate (a)

- Cartesian coordinates:

\begin{tabular}{cccc}
\hline \multirow{2}{*}{ Atomic number } & \multicolumn{3}{c}{ Coordinates (Angstroms) } \\
\cline { 2 - 4 } & $\mathrm{X}$ & $\mathrm{Y}$ & $\mathrm{Z}$ \\
\hline 6 & 0.694849 & 1.211028 & -0.000001 \\
6 & -0.682028 & 1.211842 & 0.000001 \\
6 & -1.376589 & 0.000001 & 0.000001 \\
6 & -0.682020 & -1.211836 & 0.000001 \\
6 & 0.694856 & -1.211010 & -0.000000 \\
6 & 1.438289 & 0.000012 & -0.000001 \\
1 & 1.244800 & 2.144153 & -0.000002 \\
1 & -1.241355 & 2.136183 & 0.000000
\end{tabular}




$\begin{array}{lrrr}1 & -1.241340 & -2.136182 & 0.000001 \\ 1 & 1.244814 & -2.144131 & -0.000002 \\ 7 & -2.823061 & -0.000006 & 0.000000 \\ 8 & -3.408032 & 1.082533 & 0.000000 \\ 8 & -3.408022 & -1.082550 & -0.000001 \\ 8 & 2.732293 & 0.000009 & -0.000003 \\ 11 & 4.718223 & -0.000013 & 0.000002\end{array}$

- Energies (a.u.):

Zero-point correction=

Thermal correction to Energy=

0.095770 (Hartree/Particle)

Thermal correction to Enthalpy=

0.105373

Thermal correction to Gibbs Free Energy=

0.106317

Sum of electronic and zero-point Energies $=$

0.058335

Sum of electronic and thermal Energies=

$-673.845998$

$-673.836395$

Sum of electronic and thermal Enthalpies=

$-673.835451$

Sum of electronic and thermal Free Energies=

$-673.883433$

- Vertical excitation energies and oscillator strengths (gas phase)

$\begin{array}{lcllll}\text { Excited State } & 1: & \text { Singlet-A } & 2.2590 \mathrm{eV} & 548.84 \mathrm{~nm} & \mathrm{f}=0.0018 \\ \text { Excited State } & 2: & \text { Singlet-A } & 3.3960 \mathrm{eV} & 365.09 \mathrm{~nm} & \mathrm{f}=0.0073 \\ \text { Excited State } & 3: & \text { Singlet-A } & 3.7053 \mathrm{eV} & 334.62 \mathrm{~nm} & \mathrm{f}=0.0000 \\ \text { Excited State } & 4: & \text { Singlet-A } & 3.7870 \mathrm{eV} & 327.39 \mathrm{~nm} & \mathrm{f}=0.3934 \\ \text { Excited State } & 5: & \text { Singlet-A } & 3.8597 \mathrm{eV} & 321.23 \mathrm{~nm} & \mathrm{f}=0.0000 \\ \text { Excited State } & 6: & \text { Singlet-A } & 4.1647 \mathrm{eV} & 297.70 \mathrm{~nm} & \mathrm{f}=0.0002 \\ \text { Excited State } & 7: & \text { Singlet-A } & 4.2907 \mathrm{eV} & 288.96 \mathrm{~nm} & \mathrm{f}=0.0000 \\ \text { Excited State } & 8: & \text { Singlet-A } & 4.3369 \mathrm{eV} & 285.88 \mathrm{~nm} & \mathrm{f}=0.0002 \\ \text { Excited State } & 9: & \text { Singlet-A } & 4.4772 \mathrm{eV} & 276.92 \mathrm{~nm} & \mathrm{f}=0.0000 \\ \text { Excited State } & 10: & \text { Singlet-A } & 4.4939 \mathrm{eV} & 275.89 \mathrm{~nm} & \mathrm{f}=0.0003\end{array}$

- Vertical excitation energies and oscillator strengths (aqueous phase)

$\begin{array}{llllll}\text { Excited State } & 1: & \text { Singlet-A } & 3.4481 \mathrm{eV} & 359.57 \mathrm{~nm} & \mathrm{f}=0.4637 \\ \text { Excited State } & 2: & \text { Singlet-A } & 3.7045 \mathrm{eV} & 334.69 \mathrm{~nm} & \mathrm{f}=0.0000 \\ \text { Excited State } & 3: & \text { Singlet-A } & 4.1086 \mathrm{eV} & 301.77 \mathrm{~nm} & \mathrm{f}=0.0000 \\ \text { Excited State } & \text { 4: } & \text { Singlet-A } & 4.2707 \mathrm{eV} & 290.31 \mathrm{~nm} & \mathrm{f}=0.0011 \\ \text { Excited State } & 5: & \text { Singlet-A } & 4.6723 \mathrm{eV} & 265.36 \mathrm{~nm} & \mathrm{f}=0.0003 \\ \text { Excited State } & 6: & \text { Singlet-A } & 4.6748 \mathrm{eV} & 265.22 \mathrm{~nm} & \mathrm{f}=0.0598 \\ \text { Excited State } & 7: & \text { Singlet-A } & 4.8951 \mathrm{eV} & 253.28 \mathrm{~nm} & \mathrm{f}=0.0006\end{array}$




$\begin{array}{lrllll}\text { Excited State } & \text { 8: } & \text { Singlet-A } & 5.2951 \mathrm{eV} & 234.15 \mathrm{~nm} & \mathrm{f}=0.0003 \\ \text { Excited State } & 9: & \text { Singlet-A } & 5.7142 \mathrm{eV} & 216.98 \mathrm{~nm} & \mathrm{f}=0.0293 \\ \text { Excited State } & 10: & \text { Singlet-A } & 5.8093 \mathrm{eV} & 213.42 \mathrm{~nm} & \mathrm{f}=0.0387\end{array}$

$\mathrm{Na}^{+}-p$-nitrophenolate (b)

- Cartesian coordinates:

\begin{tabular}{cccc}
\hline \multirow{2}{*}{ Atomic number } & \multicolumn{3}{c}{ Coordinates (Angstroms) } \\
\cline { 2 - 4 } & $\mathrm{X}$ & $\mathrm{Y}$ & $\mathrm{Z}$ \\
\hline 6 & 2.022670 & 1.240927 & 0.000000 \\
6 & 0.671758 & 1.238804 & -0.000000 \\
6 & -0.045943 & 0.000000 & -0.000001 \\
6 & 0.671758 & -1.238804 & -0.000001 \\
6 & 2.022670 & -1.240927 & -0.000000 \\
6 & 2.803508 & -0.000000 & 0.000000 \\
1 & 2.585438 & 2.165493 & 0.000001 \\
1 & 0.103547 & 2.157680 & -0.000001 \\
1 & 0.103546 & -2.157680 & -0.000001 \\
1 & 2.585438 & -2.165493 & 0.000000 \\
7 & -1.394427 & 0.000000 & -0.000001 \\
8 & -2.064644 & 1.100138 & -0.000001 \\
8 & -2.064644 & -1.100138 & -0.000000 \\
8 & 4.034857 & -0.000000 & 0.000001 \\
11 & -3.976371 & -0.000000 & 0.000001
\end{tabular}

- Energies (a.u.):

Zero-point correction $=$

0.095694 (Hartree/Particle)

Thermal correction to Energy=

0.105077

Thermal correction to Enthalpy=

0.106021

Thermal correction to Gibbs Free Energy=

0.060107

Sum of electronic and zero-point Energies $=$

$-673.850914$

Sum of electronic and thermal Energies=

Sum of electronic and thermal Enthalpies=

$-673.840587$

Sum of electronic and thermal Free Energies=

$-673.886501$ 
- Vertical excitation energies and oscillator strengths (gas phase)

$\begin{array}{lccccc}\text { Excited State } & 1: & \text { Singlet-A } & 1.4250 \mathrm{eV} & 870.09 \mathrm{~nm} & \mathrm{f}=0.0017 \\ \text { Excited State } & 2: & \text { Singlet-A } & 2.1714 \mathrm{eV} & 570.98 \mathrm{~nm} & \mathrm{f}=0.0000 \\ \text { Excited State } & 3: & \text { Singlet-A } & 2.7189 \mathrm{eV} & 456.01 \mathrm{~nm} & \mathrm{f}=0.0000 \\ \text { Excited State } & 4: & \text { Singlet-A } & 3.1844 \mathrm{eV} & 389.34 \mathrm{~nm} & \mathrm{f}=0.0000 \\ \text { Excited State } & 5: & \text { Singlet-A } & 3.3610 \mathrm{eV} & 368.89 \mathrm{~nm} & \mathrm{f}=0.4473 \\ \text { Excited State } & 6: & \text { Singlet-A } & 3.5576 \mathrm{eV} & 348.50 \mathrm{~nm} & \mathrm{f}=0.0001 \\ \text { Excited State } & 7: & \text { Singlet-A } & 3.7602 \mathrm{eV} & 329.73 \mathrm{~nm} & \mathrm{f}=0.0001 \\ \text { Excited State } & 8: & \text { Singlet-A } & 3.8958 \mathrm{eV} & 318.25 \mathrm{~nm} & \mathrm{f}=0.1822 \\ \text { Excited State } & 9: & \text { Singlet-A } & 4.0555 \mathrm{eV} & 305.72 \mathrm{~nm} & \mathrm{f}=0.0009 \\ \text { Excited State } & 10: & \text { Singlet-A } & 4.0945 \mathrm{eV} & 302.81 \mathrm{~nm} & \mathrm{f}=0.0006\end{array}$

- Vertical excitation energies and oscillator strengths (aqueous phase)

$\begin{array}{lcllll}\text { Excited State } & 1: & \text { Singlet-A } & 3.2615 \mathrm{eV} & 380.15 \mathrm{~nm} & \mathrm{f}=0.0000 \\ \text { Excited State } & 2: & \text { Singlet-A } & 3.4726 \mathrm{eV} & 357.03 \mathrm{~nm} & \mathrm{f}=0.6103 \\ \text { Excited State } & 3: & \text { Singlet-A } & 3.8419 \mathrm{eV} & 322.72 \mathrm{~nm} & \mathrm{f}=0.0019 \\ \text { Excited State } & 4: & \text { Singlet-A } & 4.2735 \mathrm{eV} & 290.12 \mathrm{~nm} & \mathrm{f}=0.0017 \\ \text { Excited State } & 5: & \text { Singlet-A } & 4.6503 \mathrm{eV} & 266.62 \mathrm{~nm} & \mathrm{f}=0.0486 \\ \text { Excited State } & 6: & \text { Singlet-A } & 4.8097 \mathrm{eV} & 257.78 \mathrm{~nm} & \mathrm{f}=0.0010 \\ \text { Excited State } & 7: & \text { Singlet-A } & 4.8886 \mathrm{eV} & 253.62 \mathrm{~nm} & \mathrm{f}=0.0033 \\ \text { Excited State } & 8: & \text { Singlet-A } & 5.1537 \mathrm{eV} & 240.57 \mathrm{~nm} & \mathrm{f}=0.0004 \\ \text { Excited State } & 9: & \text { Singlet-A } & 5.6806 \mathrm{eV} & 218.26 \mathrm{~nm} & \mathrm{f}=0.0969 \\ \text { Excited State } & 10: & \text { Singlet-A } & 5.8357 \mathrm{eV} & 212.46 \mathrm{~nm} & \mathrm{f}=0.0080\end{array}$

\section{$\underline{K^{+}-p-\text { nitrophenolate }}$}

$\mathrm{K}^{+}-p$-nitrophenolate (a)

- Cartesian coordinates:

\begin{tabular}{crrc}
\hline \multirow{2}{*}{ Atomic number } & \multicolumn{3}{c}{ Coordinates (Angstroms) } \\
\cline { 2 - 4 } & \multicolumn{1}{c}{$\mathrm{X}$} & $\mathrm{Y}$ & $\mathrm{Z}$ \\
\hline 6 & 0.205200 & 1.211421 & 0.000002 \\
6 & -1.170838 & 1.212043 & 0.000001 \\
6 & -1.867212 & 0.000000 & 0.000000 \\
6 & -1.170838 & -1.212043 & 0.000001 \\
6 & 0.205200 & -1.211421 & 0.000001 \\
& 44 & & \\
\end{tabular}




$\begin{array}{rrrr}6 & 0.953691 & -0.000000 & 0.000002 \\ 1 & 0.754606 & 2.145106 & 0.000003 \\ 1 & -1.730444 & 2.136337 & 0.000001 \\ 1 & -1.730444 & -2.136337 & -0.000000 \\ 1 & 0.754606 & -2.145107 & 0.000001 \\ 7 & -3.310778 & -0.000000 & -0.000001 \\ 8 & -3.897662 & 1.082768 & -0.000003 \\ 8 & -3.897662 & -1.082768 & -0.000001 \\ 8 & 2.244555 & -0.000000 & 0.000002 \\ 19 & 4.558002 & 0.000000 & -0.000002\end{array}$

- Energies (a.u.):

Zero-point correction=

Thermal correction to Energy=

0.095434 (Hartree/Particle)

Thermal correction to Enthalpy=

0.105167

0.106111

Thermal correction to Gibbs Free Energy=

0.057372

Sum of electronic and zero-point Energies=

$-1111.489740$

Sum of electronic and thermal Energies=

$-1111.480007$

Sum of electronic and thermal Enthalpies=

$-1111.479063$

Sum of electronic and thermal Free Energies=

$-1111.527802$

- Vertical excitation energies and oscillator strengths (gas phase)

$\begin{array}{llllll}\text { Excited State } & 1: & \text { Singlet-A } & 2.4905 \mathrm{eV} & 497.83 \mathrm{~nm} & \mathrm{f}=0.0008 \\ \text { Excited State } & \text { 2: } & \text { Singlet-A } & 3.5863 \mathrm{eV} & 345.72 \mathrm{~nm} & \mathrm{f}=0.0047 \\ \text { Excited State } & \text { 3: } & \text { Singlet-A } & 3.7578 \mathrm{eV} & 329.94 \mathrm{~nm} & \mathrm{f}=0.3964 \\ \text { Excited State } & \text { 4: } & \text { Singlet-A } & 3.8538 \mathrm{eV} & 321.72 \mathrm{~nm} & \mathrm{f}=0.0000 \\ \text { Excited State } & \text { 5: } & \text { Singlet-A } & 3.9779 \mathrm{eV} & 311.69 \mathrm{~nm} & \mathrm{f}=0.0000 \\ \text { Excited State } & 6: & \text { Singlet-A } & 4.3060 \mathrm{eV} & 287.93 \mathrm{~nm} & \mathrm{f}=0.0000 \\ \text { Excited State } & 7: & \text { Singlet-A } & 4.3182 \mathrm{eV} & 287.12 \mathrm{~nm} & \mathrm{f}=0.0001 \\ \text { Excited State } & 8: & \text { Singlet-A } & 4.3962 \mathrm{eV} & 282.03 \mathrm{~nm} & \mathrm{f}=0.0000 \\ \text { Excited State } & 9: & \text { Singlet-A } & 4.4072 \mathrm{eV} & 281.32 \mathrm{~nm} & \mathrm{f}=0.0001 \\ \text { Excited State } & 10: & \text { Singlet-A } & 4.4705 \mathrm{eV} & 277.34 \mathrm{~nm} & \mathrm{f}=0.0002\end{array}$

- Vertical excitation energies and oscillator strengths (aqueous phase)

$\begin{array}{llllll}\text { Excited State } & \text { 1: } & \text { Singlet-A } & 3.4376 \mathrm{eV} & 360.67 \mathrm{~nm} & \mathrm{f}=0.4800 \\ \text { Excited State } & \text { 2: } & \text { Singlet-A } & 3.6471 \mathrm{eV} & 339.95 \mathrm{~nm} & \mathrm{f}=0.0000 \\ \text { Excited State } & \text { 3: } & \text { Singlet-A } & 4.0946 \mathrm{eV} & 302.80 \mathrm{~nm} & \mathrm{f}=0.0000 \\ \text { Excited State } & \text { 4: } & \text { Singlet-A } & 4.2753 \mathrm{eV} & 290.00 \mathrm{~nm} & \mathrm{f}=0.0009\end{array}$


Excited State $\quad$ 5: $\quad$ Singlet-A $\quad 4.6483 \mathrm{eV} \quad 266.73 \mathrm{~nm} \quad \mathrm{f}=0.0588$

Excited State 6: $\quad$ Singlet-A $\quad 4.6768 \mathrm{eV} \quad 265.10 \mathrm{~nm} \quad \mathrm{f}=0.0003$

Excited State 7: $\quad$ Singlet-A $\quad 4.8245 \mathrm{eV} \quad 256.99 \mathrm{~nm} \quad \mathrm{f}=0.0005$

Excited State 8: $\quad$ Singlet-A $\quad 5.2422 \mathrm{eV} \quad 236.51 \mathrm{~nm} \quad \mathrm{f}=0.0004$

Excited State 9: $\quad$ Singlet-A $\quad 5.6302 \mathrm{eV} \quad 220.21 \mathrm{~nm} \quad \mathrm{f}=0.0227$

Excited State 10: $\quad$ Singlet-A $\quad 5.8527 \mathrm{eV} \quad 211.84 \mathrm{~nm} \quad \mathrm{f}=0.0407$

$\mathrm{K}^{+}-p$-nitrophenolate (b)

- Cartesian coordinates:

\begin{tabular}{lccc}
\hline \multirow{2}{*}{ Atomic number } & \multicolumn{3}{c}{ Coordinates (Angstroms) } \\
\cline { 2 - 4 } & $\mathrm{X}$ & $\mathrm{Y}$ & $\mathrm{Z}$ \\
\hline 6 & 0.000000 & -2.464318 & 1.238586 \\
6 & 0.000000 & -1.111695 & 1.235788 \\
6 & 0.000000 & -0.393649 & -0.000000 \\
6 & 0.000000 & -1.111695 & -1.235788 \\
6 & 0.000000 & -2.464318 & -1.238586 \\
6 & 0.000000 & -3.245972 & -0.000000 \\
1 & 0.000000 & -3.025667 & 2.164187 \\
1 & 0.000001 & -0.544561 & 2.155453 \\
1 & 0.000001 & -0.544561 & -2.155453 \\
1 & 0.000000 & -3.025667 & -2.164187 \\
7 & 0.000000 & 0.961759 & 0.000000 \\
8 & 0.000000 & 1.622315 & 1.098573 \\
8 & 0.000000 & 1.622315 & -1.098573 \\
8 & -0.000001 & -4.479221 & -0.000000 \\
19 & 0.000000 & 3.949198 & 0.000000
\end{tabular}

- Energies (a.u.):

Zero-point correction=

0.095194 (Hartree/Particle)

Thermal correction to Energy=

0.104878

Thermal correction to Enthalpy=

0.105822

Thermal correction to Gibbs Free Energy=

0.058283

Sum of electronic and zero-point Energies=

$-1111.491647$ 
Sum of electronic and thermal Energies=

$-1111.481963$

Sum of electronic and thermal Enthalpies=

- Vertical excitation energies and oscillator strengths (gas phase)

$\begin{array}{lcllll}\text { Excited State } & \text { 1: } & \text { Singlet-A } & 2.7611 \mathrm{eV} & 449.04 \mathrm{~nm} & \mathrm{f}=0.0000 \\ \text { Excited State } & \text { 2: } & \text { Singlet-A } & 2.8320 \mathrm{eV} & 437.80 \mathrm{~nm} & \mathrm{f}=0.0019 \\ \text { Excited State } & \text { 3: } & \text { Singlet-A } & 3.5674 \mathrm{eV} & 347.55 \mathrm{~nm} & \mathrm{f}=0.0000 \\ \text { Excited State } & \text { 4: } & \text { Singlet-A } & 3.6103 \mathrm{eV} & 343.41 \mathrm{~nm} & \mathrm{f}=0.6207 \\ \text { Excited State } & 5: & \text { Singlet-A } & 4.0688 \mathrm{eV} & 304.72 \mathrm{~nm} & \mathrm{f}=0.0000 \\ \text { Excited State } & 6: & \text { Singlet-A } & 4.1236 \mathrm{eV} & 300.67 \mathrm{~nm} & \mathrm{f}=0.0006 \\ \text { Excited State } & 7: & \text { Singlet-A } & 4.5659 \mathrm{eV} & 271.54 \mathrm{~nm} & \mathrm{f}=0.0000 \\ \text { Excited State } & 8: & \text { Singlet-A } & 4.6065 \mathrm{eV} & 269.15 \mathrm{~nm} & \mathrm{f}=0.0071 \\ \text { Excited State } & \text { 9: } & \text { Singlet-A } & 4.6623 \mathrm{eV} & 265.93 \mathrm{~nm} & \mathrm{f}=0.0000 \\ \text { Excited State } & 10: & \text { Singlet-A } & 4.7654 \mathrm{eV} & 260.17 \mathrm{~nm} & \mathrm{f}=0.0000\end{array}$

- Vertical excitation energies and oscillator strengths (aqueous phase)

$\begin{array}{lrlrrr}\text { Excited State } & \text { 1: } & \text { Singlet-A" } & 3.2002 \mathrm{eV} & 387.43 \mathrm{~nm} & \mathrm{f}=0.0000 \\ \text { Excited State } & \text { 2: } & \text { Singlet-A' } & 3.5098 \mathrm{eV} & 353.26 \mathrm{~nm} & \mathrm{f}=0.6670 \\ \text { Excited State } & \text { 3: } & \text { Singlet-A" } & 3.8132 \mathrm{eV} & 325.14 \mathrm{~nm} & \mathrm{f}=0.0000 \\ \text { Excited State } & \text { 4: } & \text { Singlet-A" } & 4.2481 \mathrm{eV} & 291.86 \mathrm{~nm} & \mathrm{f}=0.0018 \\ \text { Excited State } & \text { 5: } & \text { Singlet-A' } & 4.7076 \mathrm{eV} & 263.37 \mathrm{~nm} & \mathrm{f}=0.0004 \\ \text { Excited State } & 6: & \text { Singlet-A" } & 4.7310 \mathrm{eV} & 262.07 \mathrm{~nm} & \mathrm{f}=0.0390 \\ \text { Excited State } & 7: & \text { Singlet-A' } & 5.0198 \mathrm{eV} & 246.99 \mathrm{~nm} & \mathrm{f}=0.0043 \\ \text { Excited State } & 8: & \text { Singlet-A' } & 5.2381 \mathrm{eV} & 236.69 \mathrm{~nm} & \mathrm{f}=0.0002 \\ \text { Excited State } & \text { 9: } & \text { Singlet-A" } & 5.4842 \mathrm{eV} & 226.07 \mathrm{~nm} & \mathrm{f}=0.0950 \\ \text { Excited State } & 10: & \text { Singlet-A' }^{\prime} & 5.8238 \mathrm{eV} & 212.89 \mathrm{~nm} & \mathrm{f}=0.0027\end{array}$

